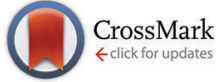

Cite this: J. Mater. Chem. B, 2015, 3, 7560

Received 24th April 2015, Accepted 21st August 2015

DOI: $10.1039 / c 5 t b 00767 d$

www.rsc.org/MaterialsB

\title{
Highly flexible silica/chitosan hybrid scaffolds with oriented pores for tissue regeneration $\uparrow$
}

\begin{abstract}
Daming Wang, ${ }^{a}$ Frederik Romer, ${ }^{\mathrm{b}}$ Louise Connell, ${ }^{a}$ Claudia Walter, ${ }^{a}$ Eduardo Saiz, ${ }^{a}$ Sheng Yue, ${ }^{c}$ Peter D. Lee, ${ }^{c}$ David S. McPhail, ${ }^{a}$ John V. Hanna ${ }^{b}$ and Julian R. Jones*a

Inorganic/organic sol-gel hybrids have nanoscale co-networks of organic and inorganic components that give them the unique potential of tailored mechanical properties and controlled biodegradation in tissue engineering applications. Here, silica/chitosan hybrid scaffolds with oriented structures were fabricated through the sol-gel method with a unidirectional freeze casting process. 3-Glycidoxypropyl trimethoxysilane (GPTMS) was used to obtain covalent inorganic/organic coupling. Process variables were investigated such as cooling rate, GPTMS and inorganic content, which can be used to tailor the mechanical properties and hybrid chemical coupling. Structural characterization and dissolution tests confirmed the covalent cross-linking of the chitosan and the silica network in hybrids. The scaffolds had a directional lamellar structure along the freezing direction and a cellular morphology perpendicular to the freezing direction. Compression testing showed that the scaffolds with $60 \mathrm{wt} \%$ organic were flexible and elastomeric perpendicular to the freezing direction whilst behaving in an elastic-brittle fashion parallel to the freezing direction. The compressive strengths are about one order of magnitude higher in the latter direction reaching values of the order of $160 \mathrm{kPa}$. This behaviour provides potential for clinicians to be able to squeeze the materials to fit tissue defect sites while providing some mechanical support from the other direction.
\end{abstract}

\section{Introduction}

Over 506 million of the global population were above 65 years of age in 2008 and this value is expected to have a $160 \%$ increase by the year of 2040. ${ }^{1}$ Increases in age-induced diseases (e.g. arthritis and osteoporosis) result in the need for the regeneration of diseased or damaged tissues. ${ }^{2}$ Tissue engineering provides a strategy for this issue. An ideal tissue regenerating scaffold must meet some critical challenges simultaneously such as being bioactive, such that it can induce new tissue ingrowth, and it should possess appropriate mechanical properties and a tailored degradation rate. ${ }^{3,4}$

Silica-based bioactive glasses fulfil most of these challenging criteria and have been successfully applied to bone tissue regeneration since they can bond to bone, degrade in the body and stimulate bone growth; however their brittle nature makes them unsuitable for defect sites that are under cyclic loading. ${ }^{5,6}$

Incorporating a biodegradable polymer to obtain inorganic/ organic composites can soften and toughen the silica matrix. ${ }^{7}$

\footnotetext{
${ }^{a}$ Department of Materials, Imperial College London, South Kensington Campus, London, SW7 2AZ, UK. E-mail: julian.r.jones@imperial.ac.uk

${ }^{b}$ Department of Physics, University of Warwick, Coventry CV4 7AL, UK

${ }^{c}$ Manchester X-ray Imaging Facility, School of Materials, University of Manchester, Oxford Road, Manchester M13 9PL, UK

$\dagger$ Electronic supplementary information (ESI) available. See DOI: 10.1039/c5tb00767d
}

Conventional inorganic/organic composites have two distinguishable phases interacting at the micrometre scale and as a result, bioactive glass particles (bioactive phase) can be enclosed in the polymer matrix (non-bioactive phase) which could lead to poor cell response. ${ }^{4}$ The two different phases also cause different degradation behaviours and therefore scaffold instability and glass particle migration in vivo. ${ }^{8}$

Cartilage performs as a shock absorber and a good bearing surface due to the organization of collagen fibrils and proteoglycan molecules. It has zones: articular, middle, and deep zones, each with different collagen fibril orientation. ${ }^{9}$ Current repair methods involve drilling holes into the bone marrow to free stem cells, which as sometimes kept in position with the use of hydrogels. ${ }^{10}$ Scaffolds that can assist with organization of the cartilage are needed.

The creation of sol-gel inorganic/organic hybrid materials has potential to tackle the aforementioned problems as the inorganic and organic components form co-networks at the molecular level, providing congruent degradation. ${ }^{4,11-13}$ In class II hybrids, covalent coupling is obtained between the organic and inorganic networks. The hybrids can retain bioactivity from bioactive glasses since host tissue/cells can contact both inorganic and organic components simultaneously as if it is one material. ${ }^{4}$

Chitosan is a natural polysaccharide that has been widely used in biomedical field ${ }^{14,15}$ and was used to synthesize 
inorganic/organic hybrids with 3-glycidoxypropyltrimethoxysilane (GPTMS), which contains an epoxide ring on one end and three methoxysilane groups on the other. ${ }^{16-18}$ For most of these hybrid materials GPTMS was the only inorganic component, which means adjusting the GPTMS content to alter the inorganic/organic coupling degree would also change the inorganic/organic ratios simultaneously. Independent control of composition is needed. Shirosaki et al. ${ }^{19}$ and Connell et al. ${ }^{20}$ introduced functionalized chitosan into the tetraethylorthosilicate (TEOS) based sol-gel process, using GPTMS as a coupling agent between the sol-gel silica and the chitosan. GPTMS was hypothesized to functionalize chitosan through the nucleophilic attack of the epoxide ring of the GPTMS by the primary amine group $\left(-\mathrm{NH}_{2}\right)$ of the chitosan. ${ }^{18,21}$ The functionalized chitosan would then have methoxysilane groups, which on addition to the sol would hydrolyse forming -Si-OH groups that can undergo polycondensation with $-\mathrm{Si}-\mathrm{OH}$ groups from the hydrolysed TEOS, forming Class II silica/chitosan hybrids. ${ }^{20}$ The aim of having a separate silica source (TEOS) and using GPTMS as a coupling agent, was that mechanical properties and degradation rates can be tailored by controlling the inorganic/organic ratio and the coupling degree (molar ratio of the polymer and GPTMS). ${ }^{11,20,22,23}$

For tissue engineering, three dimensional scaffolds with interconnected pore networks and sufficient pore sizes are required for tissue ingrowth. Porous GPTMS/chitosan hybrid scaffolds were produced previously from the freeze drying technique had approximately isotropic microstructures. ${ }^{17,20}$ However, the structures of various tissues (e.g. sub-articular cartilage and skeletal muscle) are complex and orientation of the pore networks varies from site to site.

The development of freeze casting provides a strategy to obtain porous scaffolds with aligned pores and the structure can yield anisotropic mechanical response, where a scaffold should be stronger and stiffer along the freezing direction. ${ }^{24-28}$ Mechanical properties and pore sizes can be tailored by adjusting several parameters during freeze casting such as freezing rate i.e. higher cooling rate leads to finer structures and therefore higher compressive strength along the freezing direction, and temperature gradient direction, which affects the directionality of oriented pore walls. ${ }^{26,29}$

While chitosan has been freeze cast, ${ }^{30}$ we believe this is the first freeze-cast class II hybrid produced by combining the sol-gel process and the freeze casting technique. The aim was to develop the first silica/chitosan hybrid scaffolds with covalent coupling between components and anisotropic pore structures. The effect of cooling rate, TEOS and GPTMS quantities on chemical structures/cross-linking, scaffold morphology and mechanical properties of hybrid scaffolds were investigated.

\section{Materials and methods}

\subsection{Fabrication of freeze cast silica/chitosan hybrid scaffold}

All reagents were supplied by Sigma Aldrich UK unless otherwise indicated. Freeze cast silica/chitosan hybrid scaffolds were fabricated with various inorganic/organic weight ratios and
Table 1 Notation of different freeze cast scaffolds studied in this work

\begin{tabular}{lcll}
\hline Sample code & Organic (wt\%) & GC & Molar ratio of TEOS : chitosan \\
\hline 6040 GC4 & 40 & 4 & 17.59 \\
5050 GC4 & 50 & & 11.39 \\
4060 GC4 & 60 & 2 & 7.26 \\
4060 GC2 & & 1 & 2.77 \\
4060 GC 1 & & - & 6.98 \\
4060 NoGC & & - & -
\end{tabular}

molar ratios of chitosan and GPTMS (GCs), as shown in Table 1. For example, GC4 means the molar ratio of chitosan monomer: GPTMS ratio is $4: 1$. This was achieved by maintaining the chitosan concentration in solution for all the compositions whilst varying the hydrolyzed TEOS and GPTMS content added to the hybrid system. For comparison freeze cast pure chitosan scaffolds (termed FC chitosan here) and scaffolds without the addition of GPTMS (here termed NoGC) were also investigated as control groups. A list of freeze cast scaffolds with various compositions that were investigated in this work is given in Table 1. Five samples with the following compositions were studied: hybrids containing $60 \mathrm{wt} \%$ organic with different molar ratios of chitosan and GPTMS (4060 GC1, 4060 GC2 and 4060 GC4), hybrid with $50 \mathrm{wt} \%$ organic (5050 GC4) and hybrid with $40 \mathrm{wt} \%$ organic (6040 GC4). Initially a cooling rate of $10{ }^{\circ} \mathrm{C} \mathrm{m^{-1 }}$ was used.

2.1.1. Preparation of class II silica/chitosan hybrid sol. Fig. S1 (ESI $\dagger$ ) describes the synthesis steps of class II silica/chitosan hybrid sol, with the expected reaction occurring in each step. Initially $1.5 \mathrm{~g}$ chitosan powder $\left(M_{\mathrm{w}}: 50-150 \mathrm{kDa}\right.$, degree of deacetylation of $75 \%$ ) was added to deionized water to achieve a chitosan solution concentration of $\sim 17 \mathrm{mg} \mathrm{mL}{ }^{-1}$ (Fig. S1a, ESI $\dagger$ ). For dissolving the chitosan powder $2 \mathrm{~N}$ hydrochloric acid ( $\mathrm{HCl}$ ) was added drop wise to allow chitosan protonation and eventually to adjust the $\mathrm{pH}$ of the solution to 4 for further functionalization with GPTMS (Fig. S1a, ESI $\dagger$ ). Stirring was continued for $24 \mathrm{~h}$ to obtain fully dissolved chitosan solution. $\mathrm{A} \mathrm{pH}$ of 4 was selected to give a balance between reactivity and solubility and was based on the previous research. In reactions of GPTMS without chitosan, the nucleophilic reaction is acid catalyzed, but if the $\mathrm{pH}$ is too low the epoxy ring opens spontaneously in water-induced, forming diols, which prevents the nucleophilic reaction occurring. ${ }^{20,21}$ If the $\mathrm{pH}$ is above 5 the solubility of the chitosan reduces, therefore a $\mathrm{pH}$ of 4 was chosen. However for GPTMS in the presence of chitosan, Connell et al. saw the same amount of diol forming relative to the reaction regardless of $\mathrm{pH}$. The kinetics of the reaction was affected by the $\mathrm{pH}$ rather than the equilibrium. Therefore the absolute amount of diol was greater at 2 than at $4 .^{20}$

To obtain freeze cast pure chitosan scaffolds (FC chitosan) for comparison with silica/chitosan hybrid scaffolds, the chitosan solution was directly transferred to the freeze caster at this point.

For the synthesis of silica/chitosan hybrid sol, functionalization of chitosan and GPTMS was subsequently carried out by adding an appropriate amount of GPTMS into chitosan solution (Fig. S1b, ESI $\dagger$ ). Connell et al. reported that sufficient time was needed for the coupling reaction between chitosan and GPTMS. $^{20}$ Therefore, the sol was stirred for 3 days. A reaction 
temperature of $40{ }^{\circ} \mathrm{C}$ was chosen for functionalization, and this was driven by the observation from Gabrielli et al. that the coupling reaction between GPTMS and propanoic acid can be accelerated at elevated temperature. ${ }^{21}$ A functionalized sol with various GCs should be obtained in this step.

The inorganic content can be controlled independently by introducing a separate silica source, TEOS. TEOS was hydrolysed using an $\mathrm{R}$ ratio of 4 (the molar ratio of water to TEOS) and a water/HCl $(2 \mathrm{~N}$ ) volume ratio of 3 (Fig. S1c, ESI $\dagger$ ), based on previous work. $^{20}$ The silica sol was vigorously agitated for $1 \mathrm{~h}$ to fully hydrolyze TEOS and added to the functionalized chitosan sol, which was mixed for further $30 \mathrm{~min}$ to produce a class II silica/chitosan hybrid sol for freeze casting (Fig. S1d, ESI $\dagger$ ).

2.1.2. Freeze casting. Once the aforementioned silica/chitosan hybrid sol was obtained, a freeze casting process was applied to fabricate porous scaffolds with aligned structures. It was initiated by pouring the hybrid sol into a Teflon mould with a copper base, which was then transferred to a freeze caster and placed on the copper top of a cold finger that had an integrated thermocouple (Fig. S2, ESI $\dagger$ ). Liquid nitrogen was used for cooling down the system temperature to freeze the hybrid sol. The hybrid sol was unidirectionally frozen from the bottom of the mould (the side with the copper base) and the cold finger was cooled down from $20{ }^{\circ} \mathrm{C}$ to $-100{ }^{\circ} \mathrm{C}$ or $-120{ }^{\circ} \mathrm{C}$ at a rate of $5{ }^{\circ} \mathrm{C} \mathrm{min}{ }^{-1}$ or $10{ }^{\circ} \mathrm{C} \mathrm{min}{ }^{-1}$ for sufficient freezing, respectively. The resulting gel dwelled at the final frozen temperature for 7 minutes to ensure it was frozen solid. The frozen sample was then taken from the Teflon mould and transferred to a CoolSafe110-4 freeze-drier for a three-day drying process. The ice crystals inside the sample were thereby sublimated and lamellar structures formed.

\subsection{Evaluation of hybrid cross-linking and chemical structure}

2.2.1. Characterization of hybrid chemical structure. ${ }^{13} \mathrm{C}$ crosspolarization magic angle spinning NMR $\left({ }^{13} \mathrm{C}\right.$ CPMAS NMR $)$ was used to examine the reactions between/within chitosan and GPTMS. The ${ }^{13} \mathrm{C}$ CPMAS data were acquired on a Bruker DSX-400 spectrometer with a 9.4 T magnet operating at 100.3 MHz. The samples were spun at $10 \mathrm{kHz}$ using a Bruker $4 \mathrm{~mm} \mathrm{HX}$ probe. The initial ${ }^{1} \mathrm{H} \pi / 2$ pulse was $2.5 \mu \mathrm{s}$ and the contact time was $1 \mathrm{~ms}$. The recycle time was $3 \mathrm{~s}$ and 3072 transients were recorded from each sample for an experimental time of approximately 3 hours.

${ }^{29}$ Si magic angle spinning NMR $\left({ }^{29} \mathrm{Si}\right.$ MAS NMR) was applied to investigate the cross-linking degree of the silica network from GPTMS and TEOS. The ${ }^{29} \mathrm{Si}$ one pulse data were acquired on a Varian InfinityPlus spectrometer with a $7.05 \mathrm{~T}$ magnet operating at $56.59 \mathrm{MHz}$. The samples were spun at $5 \mathrm{kHz}$ using a Bruker $7 \mathrm{~mm}$ HX probe. The excitation pulse duration was $6 \mu$ s for a tip angle of $\sim \pi / 4$. The recycle time was $240 \mathrm{~s}$ and the acquisition time of each experiment was approximately 24 hours. The relative intensity of the silica $\mathrm{Q}^{n}$ and $\mathrm{T}^{n}$ species were determined from the deconvolution of the data and the degree of condensation $\left(D_{\mathrm{c}}\right)$ of the silica network was calculated as follows: ${ }^{20}$

$$
D_{\mathrm{c}}=\left(\frac{2 \mathrm{~T}^{2}+3 \mathrm{~T}^{3}}{3}+\frac{2 \mathrm{Q}^{2}+3 \mathrm{Q}^{3}+4 \mathrm{Q}^{4}}{4}\right) \times 100 \%
$$

A Nicolet iS10 FT-IR spectrometer (Thermo scientific) was used to obtain Fourier transform infrared spectroscopy (FTIR) spectra of freeze cast scaffolds in the attenuated total reflectance (ATR) mode. Scaffolds were ground into fine powder and dried at $40{ }^{\circ} \mathrm{C}$ prior to FTIR analysis. Spectra were obtained in the range of 500-4000 $\mathrm{cm}^{-1}$ at a resolution of $4 \mathrm{~cm}^{-1}$ and averaged over 32 scans.

Powder X-ray diffraction were collected using a PANalytical X'PERT Pro MPD X-ray diffractometer with a step size of $0.0330^{\circ}$, 200 seconds per step (10 seconds per step for the auto-slits scan), measuring between 5 and 60 degrees $2 \theta$. The radiation source was a $\mathrm{Ni}$ filtered $\mathrm{CuK} \alpha$. Automatic theta-compensating slits were used to keep the same sample area illuminated over a wide angular range.

2.2.2. Dissolution study. The dissolution behaviour of hybrid scaffolds was analysed from the soluble silica release profile in TRIS buffer solution, which was obtained using inductive coupled plasma-optical emission spectroscopy (ICP-OES, iCAP 6300, Thermo Scientific). $0.05 \mathrm{M}$ TRIS buffer solution was adjusted to $\mathrm{pH} 7.3$ at $37{ }^{\circ} \mathrm{C}$ with $2 \mathrm{~N} \mathrm{HCl}$ for mimicking a physiological $\mathrm{pH}$. The ratio of sample mass to TRIS solution volume was kept constant for all samples, which was defined by Maçon et al. ${ }^{31} 80 \mathrm{mg}$ of hybrid scaffold was immersed into $120 \mathrm{~mL}$ TRIS buffer solution. Orbital shaking was subsequently carried out at $120 \mathrm{rpm}$ at $37{ }^{\circ} \mathrm{C}$. $1 \mathrm{~mL}$ sample solution was extracted at 1, 2, 4, 8, 24, 72, $168 \mathrm{~h}$ and replaced by $1 \mathrm{~mL}$ fresh TRIS solution for compensation. Each composition was repeated in triplicate. In addition, the samples after four-week immersion were recovered by filtration, washing with deionized water and acetone and drying at $37{ }^{\circ} \mathrm{C}$ for further analysis by FTIR and thermogravimetric analysis (TGA).

2.2.3. Thermal analysis. TGA was used to determine how much chitosan remained in the scaffolds before and after fourweek immersion in TRIS buffer, thereby estimating whether the inorganic and organic components were successfully cross-linked and therefore had a congruent degradation. The samples were finely ground and dried at $40{ }^{\circ} \mathrm{C}$ to remove any moisture to a maximum level prior to the thermal analysis. TGA was carried out using a Netzsch 449 C STA instrument. Samples were placed in a platinum crucible and heated from 25 to $800{ }^{\circ} \mathrm{C}$ in air environment at a heating rate of $10 \mathrm{~K} \mathrm{~min}^{-1}$, with a blank platinum crucible as a reference.

\subsection{Hybrid scaffold morphology}

The pore morphology and microstructures of the freeze cast scaffolds fabricated at different cooling rates with various compositions were imaged by using a scanning electron microscope (SEM, JEOL JSM-5610 LV) with an accelerating voltage of 5-15 kV and working distance between 9-22 mm. All samples were stuck with carbon double-sided tapes on an aluminium die and gold coated at $20 \mathrm{~mA}$ for 2 min before imaging.

Due to the flexibility of freeze cast silica/chitosan hybrid scaffolds used in this work, it was easy to damage the hybrid microstructures during the sample sectioning step for SEM imaging. Therefore X-ray micro-computed tomography $(\mu \mathrm{CT})$ was also employed as a non-destructive technique to obtain 
three dimensional (3D) images of scaffolds. ${ }^{32}$ High resolution synchrotron-based X-ray tomographic image was obtained from the Diamond-Manchester Branchline I13 at Diamond Light Source. ${ }^{33}$ The beam was monochromated to $10 \mathrm{keV}$ and the parallel-beam setup was used with a $0.45 \mu \mathrm{m}$ effective pixel size and $\sim 1 \mu \mathrm{m}$ spatial resolution. Over the $180^{\circ}$ rotation, 1800 projections were collected at 4 seconds exposure time and normalised to tomographically reconstruct into a $3 \mathrm{D}$ volume using a filtered back projection algorithm. ${ }^{34}$

The porosity of the scaffolds was carried out on the basis of the following equation:

$$
\% \text { Porosity }=\left(1-\frac{\rho_{\text {bulk }}}{\rho_{\text {skeletal }}}\right) \times 100
$$

where $\rho_{\text {bulk }}$ is the bulk density calculated by mass and dimensional analysis and $\rho_{\text {skeletal }}$ is the skeletal density of a scaffold obtained using a helium gas pycnometry (Ultrapycnometer 1000, Quantachrome).

\subsection{Mechanical testing}

The anisotropic mechanical behaviours of freeze cast silica/ chitosan hybrid scaffolds were examined by compression testing using a Zwick/Roell Z2.5 machine with a $2 \mathrm{kN}$ load cell at a compression extension speed of $0.5 \mathrm{~mm} \mathrm{~min}^{-1}$. 5 samples were cut into a dimension of typically $20 \mathrm{~mm}$ and $18 \mathrm{~mm}$ in width and thickness, respectively.

\section{Results and discussion}

\subsection{Class II silica/chitosan hybrid scaffolds by freeze casting}

To determine whether the freeze cast silica/chitosan scaffolds fabricated in this work were class II hybrids, it was essential to understand whether chitosan was successfully covalently linked to the silica network through the coupling agent GPTMS. Since the freeze casting procedure is a physical process, it was hypothesised that the chemical structure/state would not be affected. Powder X-ray diffraction pattern (Fig. 1a) of freeze cast $50 \mathrm{wt} \%$ organic hybrid scaffold showed a characteristic broad peak of amorphous chitosan at $23.3^{\circ} 2 \theta$ and a smaller broad peak at $12.1^{\circ} 2 \theta$. There is possibly a small and broad peak at $\sim 9^{\circ} 2 \theta$, which could be due to a small crystalline phase caused by GPTMS. Previously, peaks for crystal-like GTPMS have been observed at $9^{\circ} 2 \theta$ in thin films prepared under different conditions. ${ }^{35}$ In this case the peak is small and broad so the lowtemperature freeze-casting step can therefore be assumed to not induce crystallisation that would affect the bulk mechanical properties of the scaffold.

3.1.1. Solid state NMR. ${ }^{13} \mathrm{C}$ CP-MAS NMR allowed the detection of the reactions occurring between chitosan and

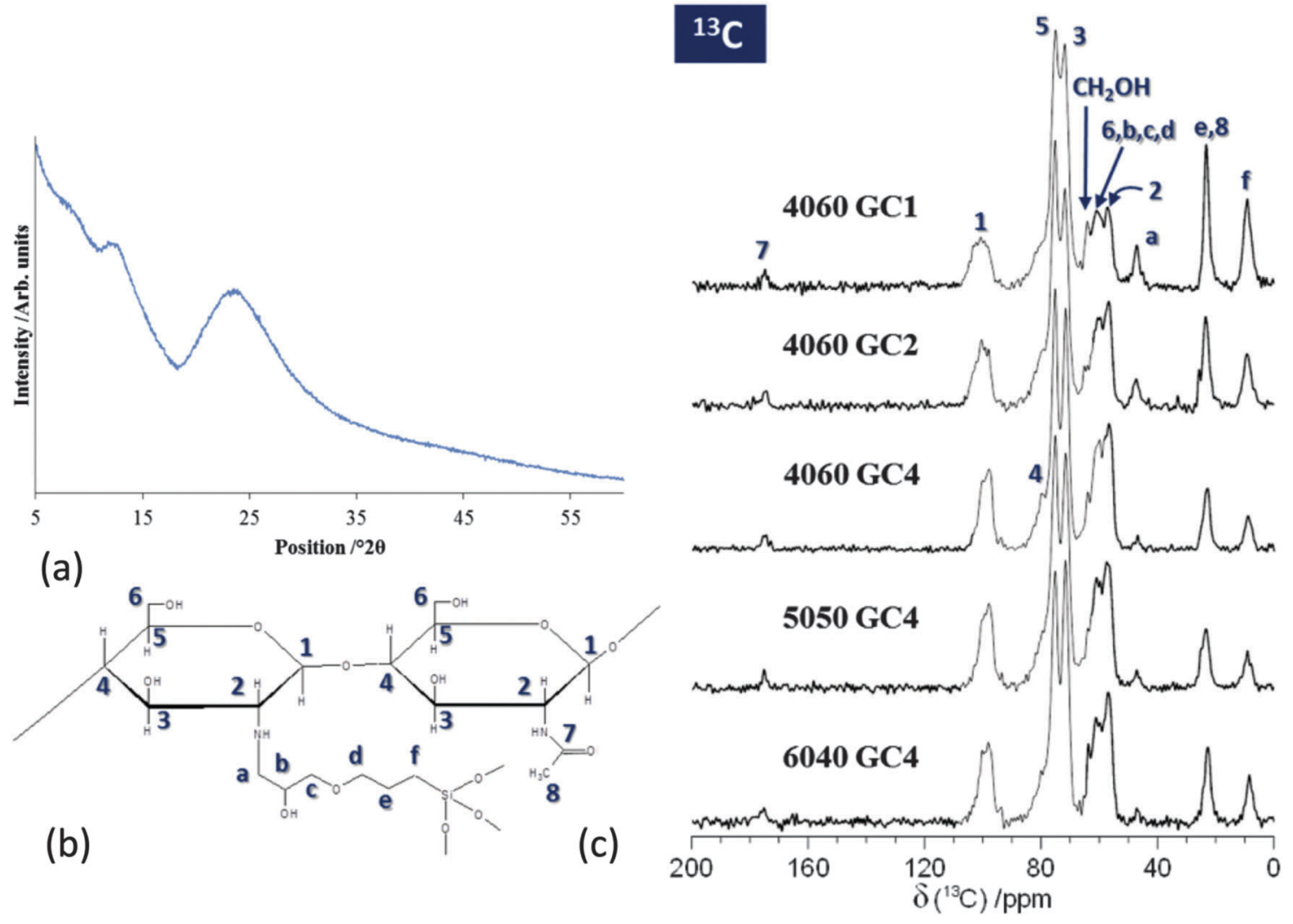

Fig. 1 (a) Powder X-ray diffraction spectrum of freeze cast silica/chitosan hybrid scaffolds with 50 wt\% organic. The small intensity of the low angle peak and the absence of any sharp diffraction peaks indicates that little crystallisation of chitosan occurs during the low-temperature freeze-casting step; (b) schematic of chitosan bonded to GPTMS (hypothesised); (c) ${ }^{13} \mathrm{C}$ CP-MAS NMR spectra of freeze cast silica/chitosan hybrid scaffolds with 60 wt\% organic of GC1 (4060 GC1), GC2 (4060 GC2) and GC4 (4060 GC4), 50 wt\% organic of GC4 (5050 GC4) and 40 wt\% organic of GC4 (6040 GC4). 
GPTMS in silica/chitosan hybrids. Fully assigned ${ }^{13} \mathrm{C}$ CP-MAS NMR data are shown in Fig. 1. The methoxy groups $\left(\mathrm{Si}-\mathrm{O}-\mathrm{CH}_{3}\right)$ associated with GPTMS ${ }^{36}$ are usually identified by a ${ }^{13} \mathrm{C}$ resonance at $\delta \sim 50 \mathrm{ppm},{ }^{36}$ however this resonance is clearly absent from these data suggesting that the hydrolysis of GPTMS to form silanol groups proceeded to completion. The resonance at $\delta \sim 64$ ppm was attributed to the formation of a diol $\left(-\left(\mathrm{CH}_{2} \mathrm{OH}\right)_{2}\right)$, which occurs due to the opening of the epoxide ring of GPTMS by water in acidic solutions. ${ }^{37,38}$ This reaction is preferential over the coupling reaction between chitosan and GPTMS. ${ }^{20}$ The ${ }^{13} \mathrm{C}$ resonance labelled ' $a$ ' (associated with the carbon in the epoxy ring) at $\delta \sim 47 \mathrm{ppm}$ shows that some epoxide rings were opened by nucleophilic attack of the primary amine of chitosan to form a secondary amine. An increase in the GPTMS content in samples GC4 to GC1 resulted in an increased intensity of resonance 'a', relative to the intensity of all chitosan resonances, suggesting chitosan/silica coupling increased. However, there was no change in the intensity of resonance 'a' relative to chitosan resonances when comparing hybrid scaffolds with different inorganic/organic ratios (i.e. 6040, 5050 and 4060 at GC4). This implies that increasing the amount of chitosan had little influence on the inorganic/organic coupling degree. While chitosan was functionalised with GPTMS, the amount of functionalization was limited and diol formation was dominant. No evidence of ethoxy groups from the TEOS were present, indicating hydrolysis was complete.

${ }^{29} \mathrm{Si}$ MAS NMR data (Fig. 2a) were acquired to quantify the degree of condensation and connectivity of the silica network structure of the silica/chitosan hybrid scaffolds. Two distinct regions can be clearly observed from $\delta \sim-50$ to $-75 \mathrm{ppm}$ and from $\delta \sim-90$ to $-120 \mathrm{ppm}$ corresponding to the silica T and Q species, respectively. The amount of $\mathrm{T}$ species indicates the degree of cross-linking between GPTMS and the silica network. The $\mathrm{T}^{n}$ denotes a silicon atom bonded to a carbon (GPTMS backbone in this case) with $n$ bridging oxygens (-Si-O-Si-). The $\mathrm{Q}$ species describe the connectivity of the silica network and the $\mathrm{Q}^{n}$ denotes a silicon with $n$ bridging oxygens (see Fig. $2 \mathrm{~b}$ ). ${ }^{39}$ Therefore, a higher value of $n$ in $\mathrm{T}$ and $\mathrm{Q}$ species indicates a more connected silica network, e.g. a silica network containing only $\mathrm{T}^{3}$ and $\mathrm{Q}^{4}$ structures represents a fully condensed network.

From the ${ }^{29} \mathrm{Si}$ MAS NMR spectra (Fig. 2a) it is clear that the $\mathrm{Q}^{3}$ and $\mathrm{Q}^{4}$ structures were the dominant $\mathrm{Q}$ species in all hybrid scaffolds with no $\mathrm{Q}^{1}$ species detected, implying a highly condensed silica network. The absence of $\mathrm{T}^{1}$ species indicates that the silica portion of the GPTMS molecules underwent a high degree of condensation. An increase in the GPTMS content from GC4 to GC1 caused significant concomitant increase in the intensity of both the $\mathrm{T}^{2}$ and $\mathrm{T}^{3}$ species, and the intensity of the resonance due to the $\mathrm{Q}^{4}$ structure was also larger. The relative intensity of $\mathrm{Q}^{4}$ was lower than that of $\mathrm{Q}^{3}$ in the GC4 scaffolds, but it slightly exceeded the intensity of the $\mathrm{Q}^{3}$ species when the molar ratio of chitosan and GPTMS reached 2 (GC2). Eventually the $\mathrm{Q}^{4}$ intensity surpassed the $\mathrm{Q}^{3}$ intensity in the GC1 composition.

The degree of condensation $\left(D_{\mathrm{c}}\right)$ of the silica network from GPTMS and TEOS is given in Table $2 \mathrm{~b}$. For a given inorganic/ organic coupling degree (GC4), increasing the organic content from $6040 \mathrm{GC} 4$ to $4060 \mathrm{GC} 4$ resulted in a decrease of the relative abundance of $\mathrm{Q}^{4}$ and the increase of the $\mathrm{T}$ species proportion (Fig. 2c). The intensity of $\mathrm{Q}^{4}$ dropped from $44.7 \%$ to $40.6 \%$ as the organic content increased from $40 \mathrm{wt} \%$ to $60 \mathrm{wt} \%$ (GC4, Table 2a). Conversely, the intensity of $\mathrm{T}^{3}$ species increased from $2.5 \%$ to $4.1 \%$ and $\mathrm{T}^{2}$ increased from $1.0 \%$ to $6.0 \%$ (Table $2 \mathrm{~b}$ ). The decrease of $\mathrm{Q}^{4}$ as the organic content increased could be due to less TEOS (the separate silica source) being introduced into the hybrid made with $60 \mathrm{wt} \%$ organic and an increase the disruption of the silica matrix due to less $\mathrm{Si}-\mathrm{O}-\mathrm{Si}$ bond formation. In addition, the GPTMS content was proportional to the amount of chitosan with a certain GC. Therefore, the 4060 GC4 hybrid scaffold had more GPTMS incorporated than 6040 GC4, causing more of the silica network to form from the silica in the GPTMS, increasing the proportion of $\mathrm{T}$ species. However, the condensation degree $\left(D_{\mathrm{c}}\right)$ of the silica network from GPTMS and TEOS were similar in all three compositions, i.e. $84.6 \%$ for $6040 \mathrm{GC} 4,83.9 \%$ for $5050 \mathrm{GC} 4$ and $84.1 \%$ for $4060 \mathrm{GC} 4$ (Table 2b), suggesting that the influence of the inorganic/organic ratios was minor on the connectivity of the silica network in this case.

For a given inorganic/organic ratio (e.g. $60 \mathrm{wt} \%$ organic), the relative proportions of $\mathrm{T}$ species increased as GPTMS incorporation increased (Fig. 2c). $\mathrm{T}^{2}$ increased from 6.0 to $14.4 \%$ as GPTMS increased from GC4 to GC1 whilst $\mathrm{T}^{3}$ increased from $4.1 \%$ to $17.1 \%$ (Table $2 \mathrm{~b}$ ), as expected. However, it is not clear whether the $\mathrm{T}^{3}$ species form by coupling to a silicate network that originates solely from the TEOS or whether other GPTMS molecules are also involved. The 4060 GC1 hybrid scaffold had a similar proportion of $\mathrm{Q}^{4}$ species $(41.6 \%)$ compared to the hybrids with GC2 and GC4 (40.6\% and 39.9\%). However, the proportion of $\mathrm{Q}^{3}$ species dropped from $43.0 \%$ to $23.6 \%$ as degree of coupling increased from GC4 to GC1 (Table 2a). As the amount of GPTMS increased the percentage of $\mathrm{T}^{3}$ and $\mathrm{T}^{2}$ species increased at the expense of $\mathrm{Q}^{3}$ species, but percentage of $\mathrm{Q}^{4}$ species remained constant. The reduction in non-bridging oxygen bonds as GPTMS content increased resulted in a more highly condensed silica network (percentage of $\mathrm{Q}^{4}+\mathrm{T}^{3}$ for GC1 was 59\% compared to $45 \%$ for GC4). This is supported by an increase of the condensation degree $\mathrm{D}_{\mathrm{c}}$ from $84.1 \%$ in GC4 to $87.7 \%$ in GC1 (Table 2b). Therefore, the amount of GPTMS has a greater influence on the condensation of the silica network in the hybrids than the inorganic/organic ratio. A more highly connected silica network was achieved by increasing the crosslinking (GPTMS content) whilst tailoring the inorganic/organic ratio showed minor effects.

3.1.2. Dissolution. The dissolution behaviour of various freeze cast silica/chitosan hybrid scaffolds in TRIS buffer was investigated. The study highlighted the effects of GPTMS quantity and the inorganic content on the degree of cross-linking of the hybrid scaffolds on dissolution. Fig. 3 shows the silicon release profiles of various freeze cast scaffolds over time.

For hybrids made with $60 \mathrm{wt} \%$ organic, silica species were released more rapidly as percentage of GPTMS decreased from the 4060 GC1 sample to 4060 NoGC. Silica release was slowest from the 4060 GC1 scaffold and the release continued until the end of the one-week dissolution test. For the 4060 GC2 and 
(a)

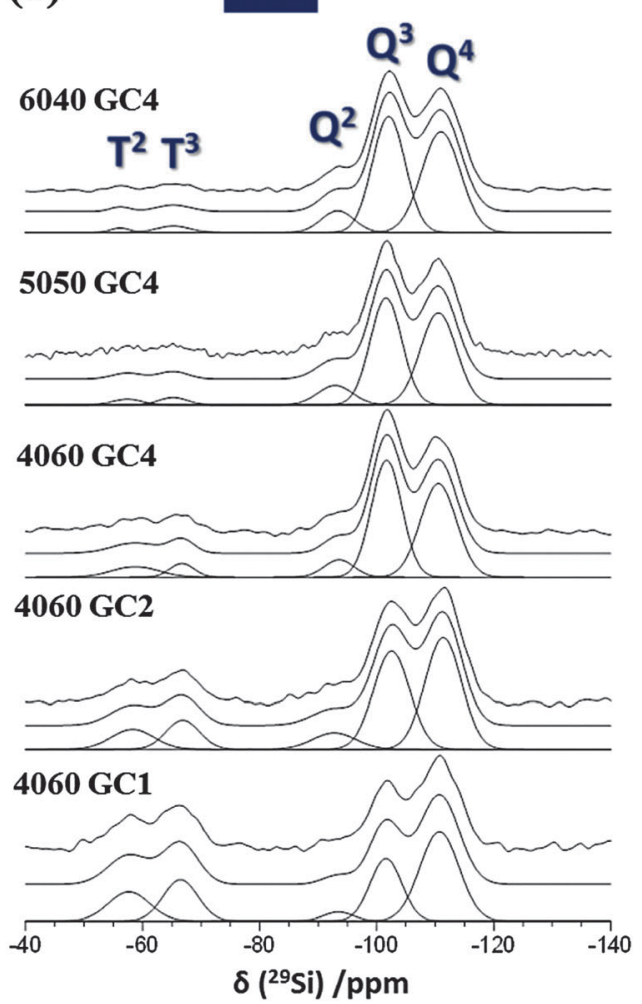

(b)

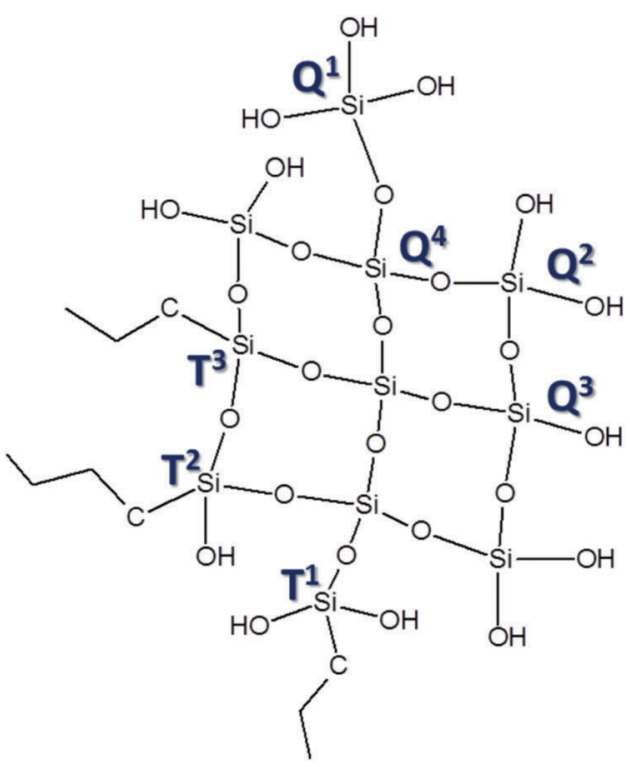

(c)

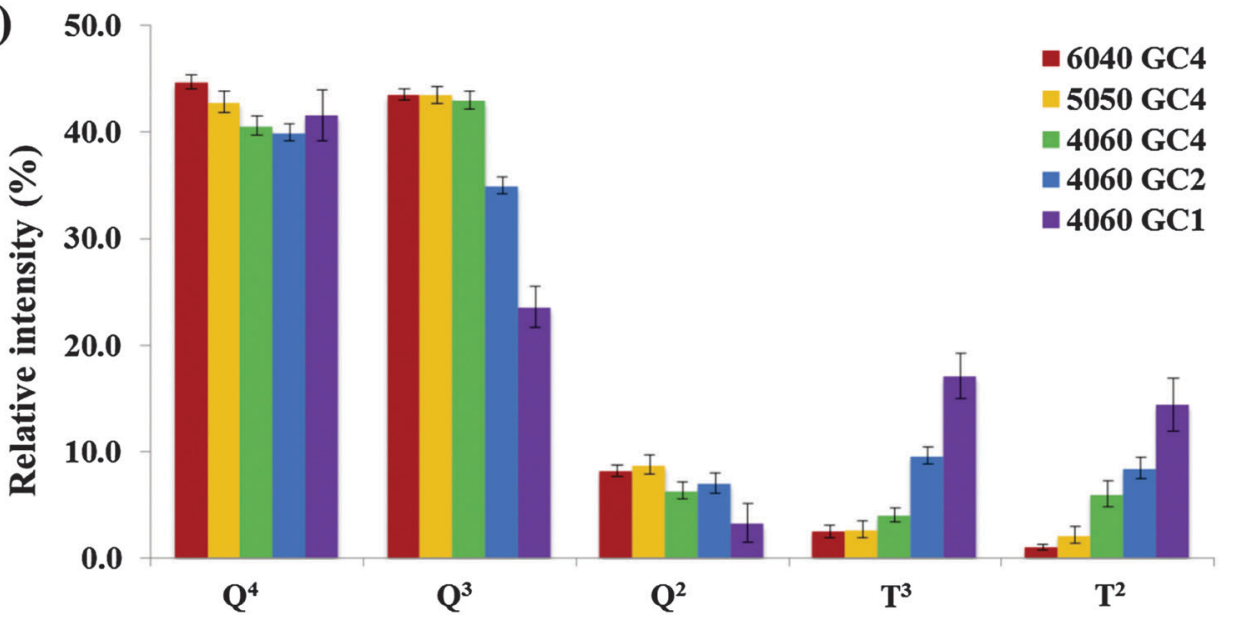

Fig. 2 (a) ${ }^{29} \mathrm{Si}$ MAS NMR spectra of freeze cast silica/chitosan hybrid scaffolds with 40 wt\% organic of GC4 (6040 GC4), 50 wt\% organic of GC4 (5050 GC4) and 60 wt\% organic of GC4 (4060 GC4), GC2 (4060 GC2) and GC1 (4060 GC1) with deconvolution also shown. (b) Schematic for the chemical structure of the silicon $T^{1}, T^{2}, T^{3}, Q^{1}, Q^{2}, Q^{3}$ and $Q^{4}$ species. (c) Relative intensity of $Q$ and $T$ species from deconvolution of the ${ }^{29}$ Si MAS NMR spectra for various freeze cast silica/chitosan hybrid scaffolds.

4060 GC4 hybrids, the silicon content of the TRIS reached a maximum and stable concentration after $72 \mathrm{~h}\left(\sim 112 \mu \mathrm{g} \mathrm{mL}{ }^{-1}\right)$ and $24 \mathrm{~h}\left(\sim 119 \mu \mathrm{g} \mathrm{mL}^{-1}\right)$, respectively. Without the addition of GPTMS for coupling, the 4060 NoGC scaffold had the maximum silica release $\left(\sim 100 \mu \mathrm{g} \mathrm{mL} \mathrm{mL}^{-1}\right)$ within only $4 \mathrm{~h}$. Therefore, the existence of GPTMS performed a critical function in tailoring the dissolution rate of the silica network. This may be due to the increasing the GPTMS content resulting in more formation of bridging oxygen silicon bonding ( $\mathrm{Si}-\mathrm{O}-\mathrm{Si})$ and therefore a more condensed silica network, as shown in solid state NMR results (Table 2). In addition, the higher proportion of the silica $\mathrm{T}$ species in the hybrids as GPTMS content increased (Fig. 2c) suggests improved inorganic/organic coupling, which can retard the scaffold dissolution rate. However, the lowest value of the final silicon concentration $\left(86 \mu \mathrm{g} \mathrm{mL}{ }^{-1}\right)$ released to the TRIS buffer after one week was for the sample without GPTMS (4060 NoGC). The presence of GPTMS therefore reduced the rate of silica release but the lowest amount of GPTMS (GC4) caused 
Table 2 Deconvolution of ${ }^{29} \mathrm{Si}$ MAS NMR spectra for freeze cast silica/chitosan hybrid scaffolds with various compositions, showing (a) the $Q$ species and (b) the T species and the condensation degree $D_{c} . \delta, \Delta$ and / represent the ${ }^{29} \mathrm{Si}$ chemical shift, the linewidth full-width half-maximum and the relative intensity, respectively

\begin{tabular}{|c|c|c|c|c|c|c|c|c|c|}
\hline (a) & \multicolumn{3}{|l|}{$\mathrm{Q}^{2}$} & \multicolumn{3}{|l|}{$\mathrm{Q}^{3}$} & \multicolumn{3}{|l|}{$\mathrm{Q}^{4}$} \\
\hline 6040 GC4 & -93.3 & 6.6 & $8.2 \pm 0.5$ & -102.1 & 6.6 & $43.5 \pm 0.5$ & -111.0 & 7.8 & $44.7 \pm 0.6$ \\
\hline 4060 GC4 & -93.6 & 6.0 & $6.3 \pm 0.8$ & -101.7 & 6.2 & $43.0 \pm 0.8$ & -110.6 & 7.3 & $40.6 \pm 0.9$ \\
\hline $4060 \mathrm{GC} 2$ & -92.7 & 8.7 & $7.0 \pm 1.0$ & -102.6 & 7.3 & $35.0 \pm 0.8$ & -111.4 & 7.3 & $39.9 \pm 0.8$ \\
\hline 4060 GC1 & -93.5 & 6.1 & $3.3 \pm 1.8$ & -101.6 & 6.4 & $23.6 \pm 1.9$ & -110.7 & 7.9 & $41.6 \pm 2.4$ \\
\hline
\end{tabular}

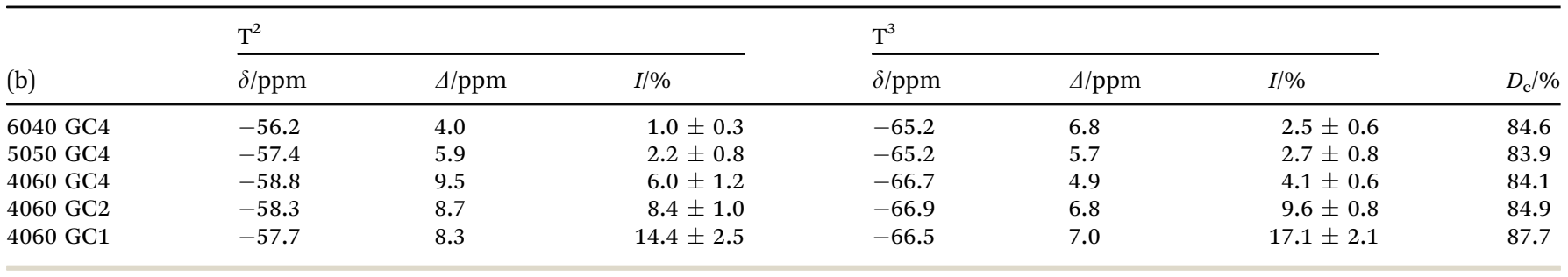

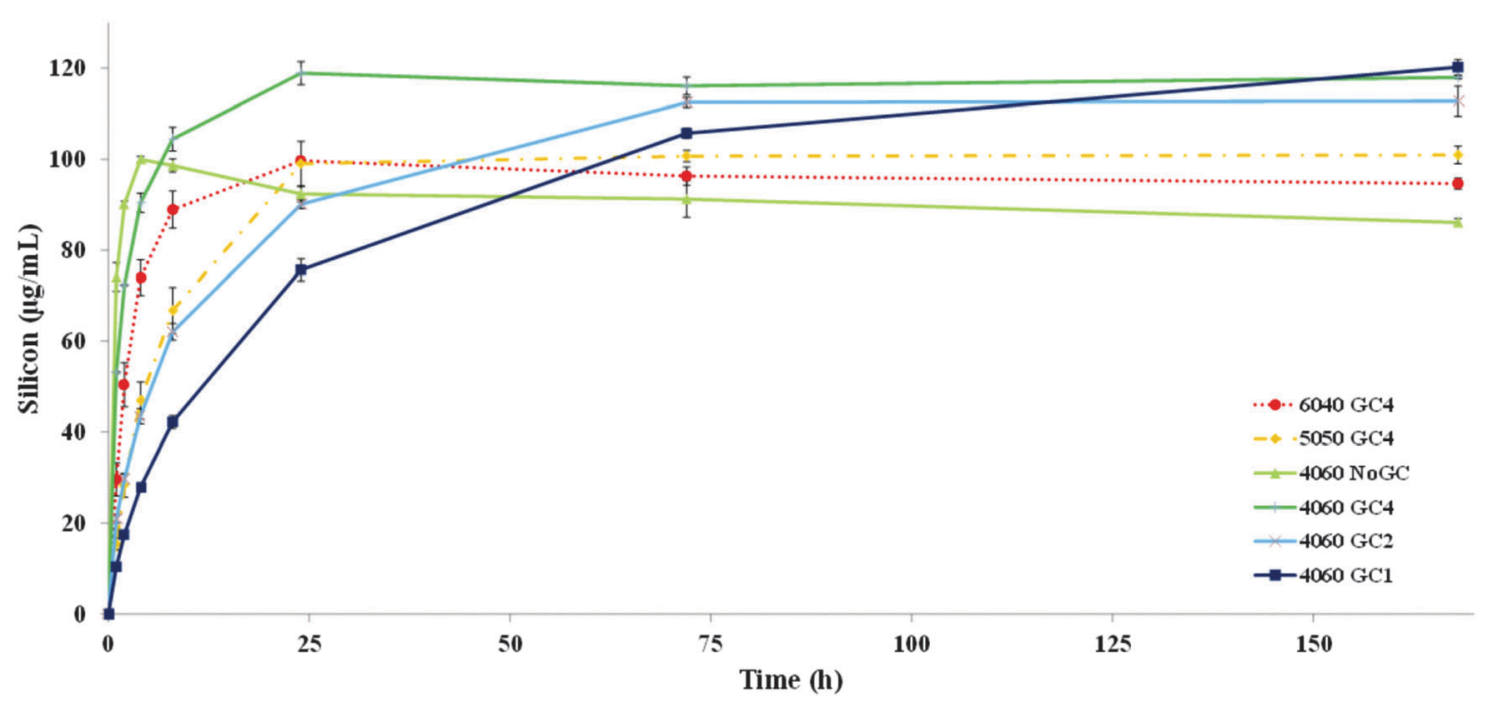

Fig. 3 Silicon release profiles in TRIS buffer solution up to one week for freeze cast hybrid scaffolds with varying GC and inorganic/organic weight ratio. The $60 \mathrm{wt} \%$ organic hybrid without the GPTMS addition (4060 NoGC) was also investigated for comparison.

an increase in the total silica released from the scaffold. Yet then as GPTMS content increased the rate of silica release reduced. A similar trend also occurred in the silica/ $\gamma$ PGA hybrid systems of various GCs and could be explained by the GPTMS disrupting the silica network without always coupling the inorganic/organic components. $^{23}$ The $\mathrm{T}^{3}$ groups could be connected together without bonding to the network that originated from the TEOS (self-condensation of hydrolysed GPTMS), so a distinct structure that originates solely from GPTMS forms, which could leave the hybrid with ease in TRIS. However, if the $\mathrm{T}$ species were self-condensing and formed a phase separate to the $\mathrm{Q}$ species, the relative intensities within the $\mathrm{Q}$ species should remain the same. This does not happen (Fig. 2a): the relative intensity of $\mathrm{Q}^{3}$ and $\mathrm{Q}^{2}$ peaks reduced with respect to $\mathrm{Q}^{4}$. This suggests that the $\mathrm{T}^{3}$ and $\mathrm{T}^{2}$ species substituted $\mathrm{Q}^{3}$ and $\mathrm{Q}^{2}$ species, during synthesis, while leaving the core $\mathrm{Q}^{4}$ relatively unaltered. GPTMS that is bonded to chitosan, but not to the silicate network, could also enter the solution with the chitosan as chitosan was released from the scaffold. ${ }^{20}$

No regular trend in the silicon release rate was found by varying the inorganic/organic weight ratio. Silica released in TRIS from the $4060 \mathrm{GC} 4$ hybrid was higher $\left(\sim 120 \mu \mathrm{g} \mathrm{mL}^{-1}\right)$ after $24 \mathrm{~h}$, compared to the other two GC4 scaffolds $\left(\sim 100 \mu \mathrm{g} \mathrm{mL} \mathrm{m}^{-1}\right.$ for both hybrids), even though it had the lowest inorganic content. This was in agreement with Connell et al. and can be explained by the disruption of the silica network due to the high organic content. $^{20}$ This suggests that an appropriate inorganic/organic ratio must be carefully considered when designing the silica/ chitosan hybrid scaffold to obtain a fully interconnected silica network. The TRIS dissolution study indicated the successful cross-linking of freeze cast silicon/chitosan hybrid scaffolds with introducing the coupling agent GPTMS, and also presented the tailorable degradation rate of such hybrids, which is promising for tissue regeneration. 
(a)

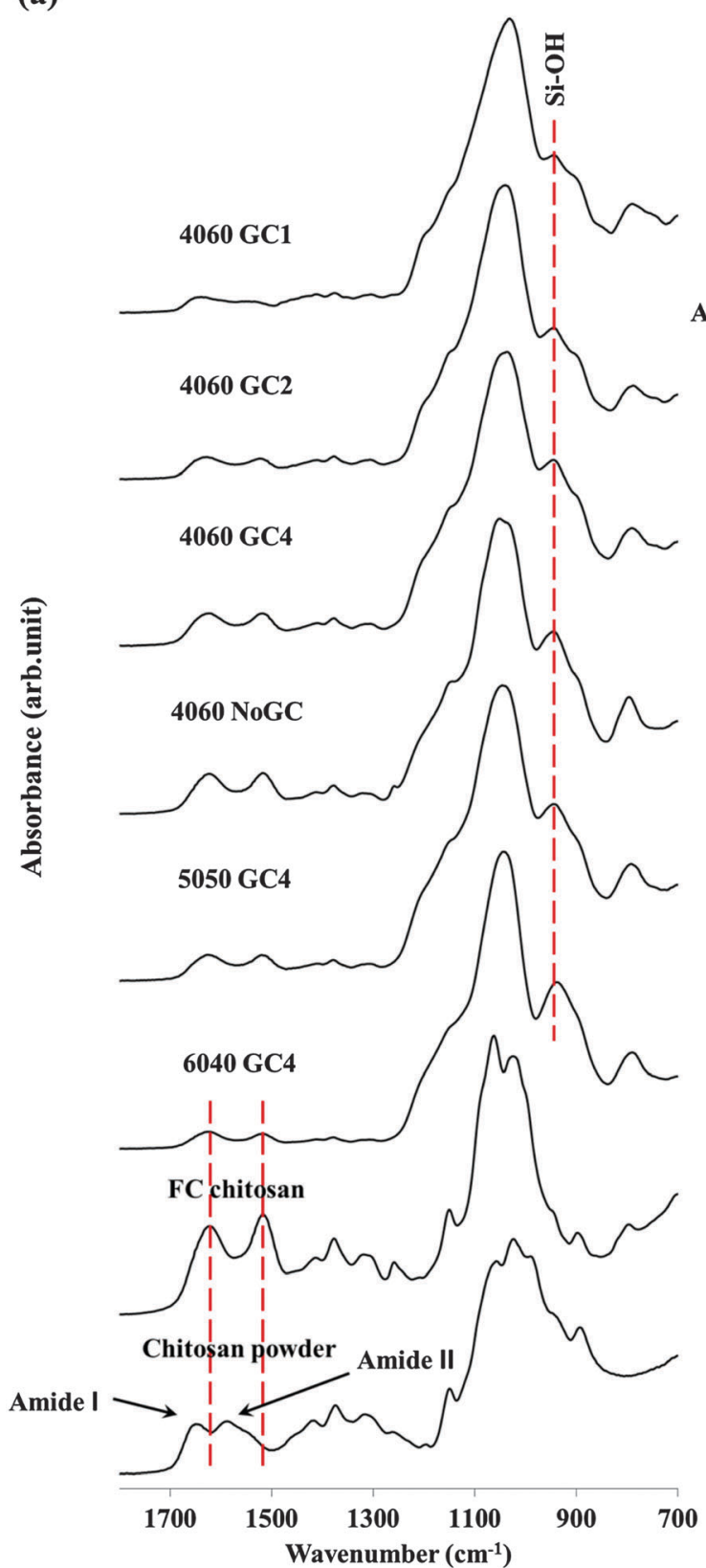

(b)

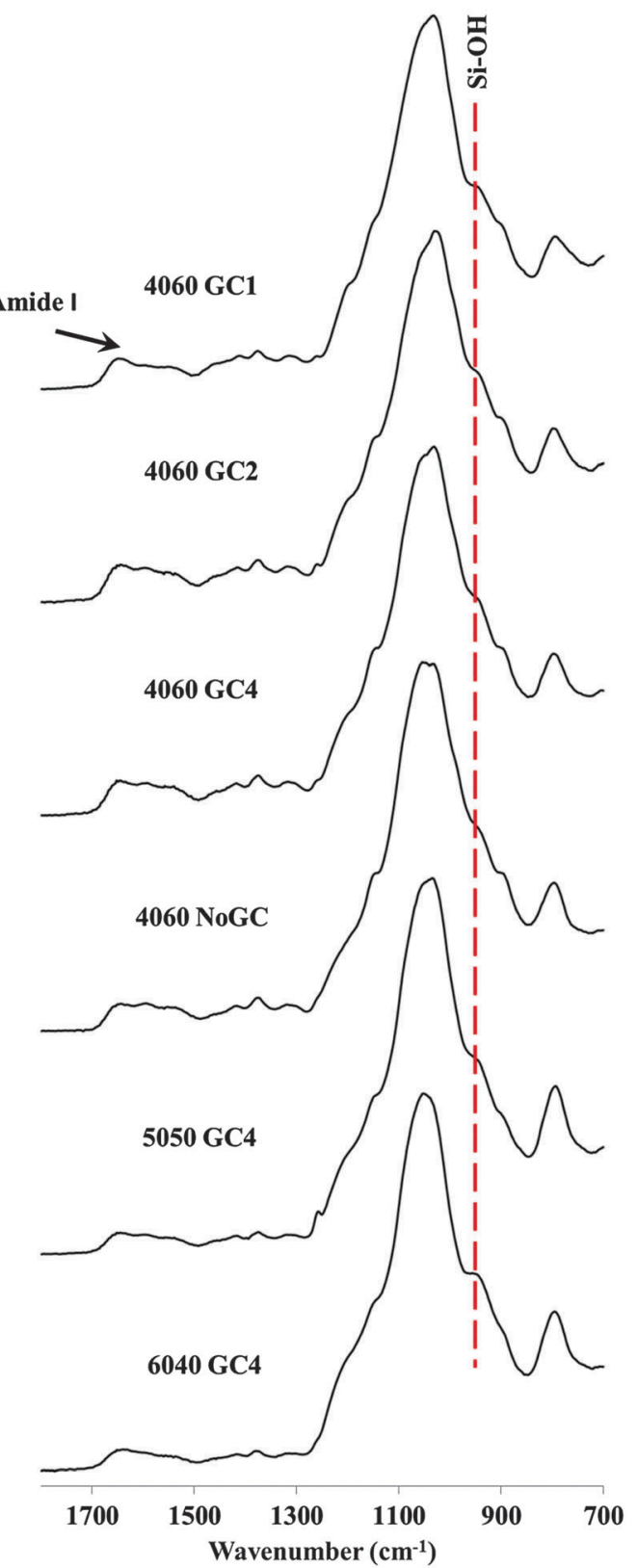

Fig. 4 FTIR spectra of (a) commercial chitosan powder and freeze cast scaffolds with various compositions and of (b) freeze cast scaffolds with various compositions after four-week immersion in TRIS buffer solution.

3.1.3. FTIR analysis. FTIR was used to determine the change in covalent bonding in freeze cast silica/chitosan hybrid scaffolds. Fig. 4a shows the FTIR spectra of freeze cast scaffolds with various compositions, in the wavenumber range of 700 to $1800 \mathrm{~cm}^{-1}$. For comparison and band assignments in hybrid scaffolds, the same commercial chitosan powder used for the hybrid synthesis and freeze cast pure chitosan scaffolds (FC chitosan) were also analysed.

An intensive band was located around $1030 \mathrm{~cm}^{-1}$ in all freeze cast hybrid scaffolds and was assigned to the stretching vibrations of the silicon bridging oxygen bonds ( $\mathrm{Si}-\mathrm{O}-\mathrm{Si}$ ), with a band at $945 \mathrm{~cm}^{-1}$ representing the non-bridging oxygens $(\mathrm{Si}-\mathrm{OH})$ in the silica network. ${ }^{11,20}$ The $\mathrm{Si}-\mathrm{OH}$ band had the lowest relative intensity for the hybrid scaffold with most GPTMS content (4060 GC1), suggesting that a more highly condensed silica network formed compared to other hybrid scaffolds. This implies increasing the GPTMS amount increased the cross-linking degree of the silica network and is in agreement with the solid state ${ }^{29} \mathrm{Si}$ NMR results (Table 2). The $\mathrm{Si}-\mathrm{OH}$ band was most prominent in the hybrid scaffolds with highest inorganic content (6040 GC4), implying that more non-bridging oxygen bonds formed in the silica network that evolved from hydrolysed TEOS rather than from the GPTMS.

Bands at 1651 and $1589 \mathrm{~cm}^{-1}$ in the spectra from the commercial chitosan powder correspond to the $\mathrm{C}=\mathrm{O}$ stretch 
of the secondary amide (amide I) in acetylated units, and the $\mathrm{N}-\mathrm{H}$ bending of the primary amine in deacetylated units (amide II), respectively. ${ }^{40,41}$ These two bands shifted to lower frequencies, i.e. to 1622 and $1518 \mathrm{~cm}^{-1}$ respectively, when the chitosan powder was dissolved and freeze cast. This phenomenon was also observed for the hybrid scaffolds. This is due to the protonation of chitosan in acidic conditions. ${ }^{42}$ The amide II band decreased as GPTMS content increased. More specifically, for the samples without GPTMS (chitosan powder, FC chitosan and 4060 NoGC) the relative intensity of the amide I bond was higher than that of amide II. The addition of GPTMS caused the relative intensity of amide II band to exceed that of amide I. This could be due to there being no reaction in the acetylated units of chitosan, whilst the chitosan reacted with GPTMS through the primary amine group $\left(\mathrm{NH}_{2}\right)$ to form a secondary amine $\left(\mathrm{C}_{\text {chitosan }}-\mathrm{NH}-\mathrm{C}_{\mathrm{GPTMS}}\right)$, resulting in the decrease in relative intensity of the amide II ( $\mathrm{N}-\mathrm{H}$ bending of the primary amine). ${ }^{43}$ Further evidence was that relative intensity of the amide II band decreased more as GPTMS increased from GC4 to GC1 and nearly disappeared in 4060 GC1. This suggests that increasing GPTMS content can promote the covalent coupling between chitosan and GPTMS and therefore the 4060 GC1 hybrid scaffold had the most inorganic/organic cross-linking reaction, which agreed with ${ }^{13} \mathrm{C}$ NMR data (Fig. 1).

Amide bands were present in all spectra at 4 weeks immersion, indicating that chitosan was still present in the hybrids (Fig. 4b). Fig. 4b showed two evident changes in covalent bonds compared to Fig. 4a, as indicated by the significant reduction in the relative intensities of the non-bridging oxygen ( $\mathrm{Si}-\mathrm{OH}$ ) band and the reduction of the amide II band for all samples.

The decrease of the non-bridging oxygen band could be due to the lower stability of the $\mathrm{Si}-\mathrm{OH}$ bond compared to that of the $\mathrm{Si}-\mathrm{O}-\mathrm{Si}$ bond; therefore, the degradation of the silica network commenced through removal of regions of silica that were bonded by few $\mathrm{Si}-\mathrm{O}-\mathrm{Si}$ bonds leaving few non-bridging oxygens after the TRIS immersion. The observation that the amide I band was still present whilst amide II was not distinguishable could be due to the dissolution of the deacetylated units (inferred from amide II due to the primary amine) in chitosan occurring preferentially over that of the acetylated units (inferred from amide I due to the $\mathrm{C}=\mathrm{O}$ bond). This could be explained by the successful cross-linking of chitosan and the silica network through the primary amine group and the reacted deacetylated units therefore dissolved with the silica, leaving the acetylated units behind.

To conclude, FTIR analysis further indicates that chitosan was successfully bonded covalently to the silica network through the coupling agent GPTMS. The results also showed a significant benefit from GPTMS to the cross-linking of inorganic and organic components, i.e. increasing the GPTMS content can promote the coupling degree of freeze cast silica/chitosan hybrid scaffolds.

3.1.4. TGA evaluation. TGA was used to measure the organic content before and after immersion in TRIS buffer for four weeks. TGA thermograms are presented in Fig. S3 (ESI $\dagger$ ) and the residual weight percentage of each material was plotted as a function of temperature. The percentages of mass change during TGA analysis are given and compared in Table 3 for each
Table 3 Percentage of mass change with quantitative values for freeze cast silica/chitosan scaffolds with various compositions before and after the four-week TRIS dissolution test. The results were obtained from the TGA data

\begin{tabular}{lllllll}
\hline & 6040 & 5050 & 4060 & 4060 & 4060 & 4060 \\
& GC4 & GC4 & NoGC & GC4 & GC2 & GC1 \\
\hline Pre TRIS & $45.0 \%$ & $54.9 \%$ & $63.7 \%$ & $63.3 \%$ & $63.5 \%$ & $61.5 \%$ \\
After TRIS & $45.2 \%$ & $56.0 \%$ & $64.9 \%$ & $72.0 \%$ & $71.0 \%$ & $67.8 \%$
\end{tabular}

composition before and after the four-week TRIS immersion. The inorganic and organic components of a class II hybrid were expected to degrade congruently in solution as they are molecular level co-networks that are covalently linked. ${ }^{11}$ The differences of the mass loss during TGA analysis of 6040 GC4 and 5050 GC4 before and after the TRIS dissolution test were similar, i.e. $45.0 \%$ (before) and $45.2 \%$ (after) for $6040 \mathrm{GC} 4$ and (54.9\% before) and $56.0 \%$ (after) for 5050 GC4. The results indicate that similar proportions of the organic to inorganic content remained in the hybrids after dissolution. This suggests that the dissolution of inorganic and organic components in 6040 GC4 and 5050 GC4 hybrids was congruent. The samples did not swell after the TRIS immersion, which may be due to the covalent interactions between the inorganic and organic components via GPTMS.

However, a significant change can be seen for the hybrid scaffolds containing $60 \mathrm{wt} \%$ organic (Table 3). As GPTMS content increased, the amount of weight loss during dissolution decreased. The increase in mass loss due to the dissolution process was $8.8 \%$ for GC4, 7.5\% for GC2 and 6.3\% for GC1. This infers that more silica was lost than chitosan in these samples during dissolution, and that less silica was lost as coupling increased. The scaffold without the GPTMS coupling (4060 NoGC) only had a minor difference before and after dissolution. Addition of GPTMS may lead to the disruption to the silica network and thus the formation of several silica network segments, ${ }^{20}$ which may further accelerate the silicon release. The ${ }^{29} \mathrm{Si}$ NMR data (Fig. 2c) support this. As the GPTMS amount increased, relative amounts of $\mathrm{T}^{3}$ and $\mathrm{T}^{2}$ increased at expense of $\mathrm{Q}^{3}$ and $\mathrm{Q}^{2}$.

The importance of selecting a suitable composition for scaffolds for tissue regeneration is hereby demonstrated, especially an appropriate inorganic/organic ratio and degree of coupling.

\subsection{Highly oriented hybrid scaffold pore structure}

One of the major features using the unidirectional freeze casting technique is to acquire a porous scaffold with oriented microstructures and tailored pore size and porosity. ${ }^{24,27,29}$ In this work the effect of composition and cooling rate on the pore morphology of freeze cast scaffolds was investigated.

Different shapes and sizes of the freeze-cast silica/chitosan hybrids can be obtained simply by using various moulds and all the scaffolds had similar pore orientation due to the unidirectional freezing from the mould base (the side directly contacted with the copper base during the freeze casting process) to the top (Fig. 5a). A cellular morphology with elongated pores can also be observed from the direction perpendicular to what the ice crystals grew (Fig. 5b). The highly ordered lamellar-like 
pores, which formed along the freezing direction, were imaged by the non-destructive $\mu \mathrm{CT}$ (Fig. 5c). The anisotropic structures have potential applications in anisotropic tissue regeneration such for sub-articular cartilage. ${ }^{44}$

To verify the pore structures obtained by freeze casting, SEM images of the scaffolds with various compositions were obtained. Fig. 6 presents the cross-sections of the scaffold structures perpendicular to the freezing direction. Although the pores of all the freeze cast scaffolds had a cellular morphology in this direction, the pore shapes varied with the inorganic and the GPTMS content. More specifically, petal-like structures formed in the freeze cast pure chitosan scaffold (FC chitosan) with elongated pores. When silica was introduced, the elongation remained in the scaffolds with high organic hybrid and low GPTMS incorporation (4060 GC4) but the pores became more angular. Increasing the silica content (from pure chitosan to 6040) and the GPTMS amount (from 4060 GC4 to 4060 GC1) led to the reduction in the degree of elongation and resulted in polygonal pores, which caused a decrease in the length of the long axis of the pores. When the increase of the inorganic/ organic weight ratio (here shown by the 5050 and 6040 hybrids) or the decrease of the molar ratio of chitosan and GPTMS (here presented by the GC2 and GC1 hybrids) exceeded a threshold, the long and short axes of the pores tended to be more similar in length.

As a consequence of the change in pore morphology, pore size comparison was challenging between compositions. In high organic scaffolds (4060 and FC chitosan) the range of pore size was also large. However, the influence of the inorganic and the GPTMS content on pore size can be estimated by focusing on the scaffolds with regular pores which were fabricated at a certain cooling rate, i.e. $5050 \mathrm{GC} 410{ }^{\circ} \mathrm{C} \mathrm{min}^{-1}$, $6040 \mathrm{GC} 410{ }^{\circ} \mathrm{C} \mathrm{min}{ }^{-1}$, $4060 \mathrm{GC} 210{ }^{\circ} \mathrm{C} \min ^{-1}$ and $4060 \mathrm{GC} 1$ $10{ }^{\circ} \mathrm{C} \mathrm{min}^{-1}$. The pore size decreased as the inorganic content increased from means of $\sim 50 \mu \mathrm{m}$ for 5050 to $<40 \mu \mathrm{m}$ for 6040 and the GPTMS amount (from $\sim 50 \mu \mathrm{m}$ for $4060 \mathrm{GC} 2$ to $\sim 40 \mu \mathrm{m}$ for $4060 \mathrm{GC} 1)$.

For FC chitosan and 4060 hybrid scaffolds the pores formed according to the anisotropic growth kinetics of the ice crystals. In this case ice grew more rapidly along the freezing direction and the crystal thickness remained small, resulting in the rodlike ice crystals and therefore the lamellar-like pores. ${ }^{26,29}$

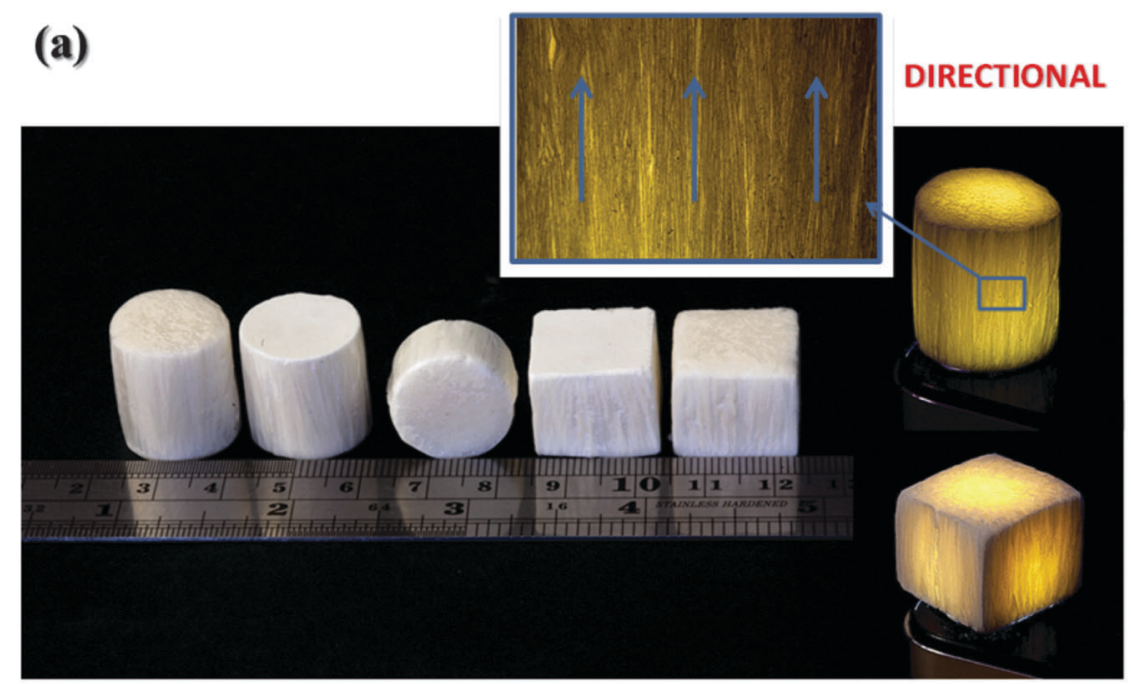

(b)

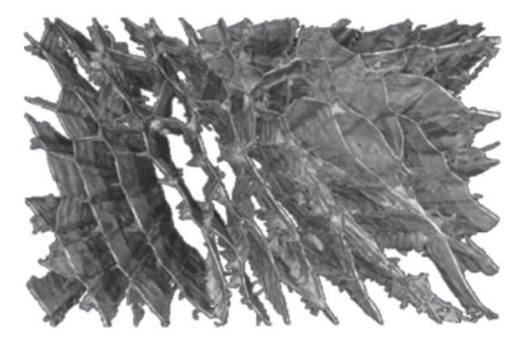

$150 \mu \mathrm{m}$ (c)

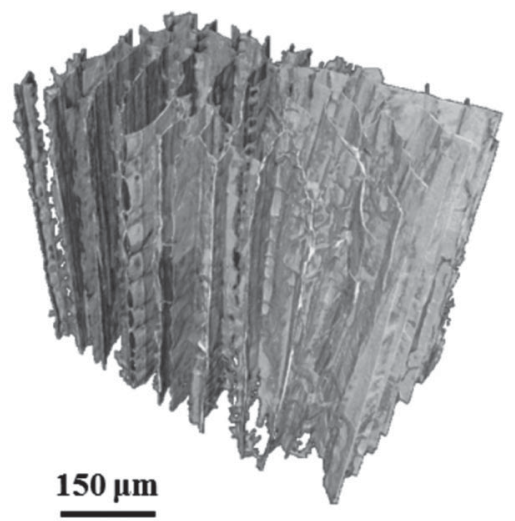

Fig. 5 (a) Images showing the freeze cast silica/chitosan hybrid scaffolds. A light source was used to obtain a clearer scaffold appearance (shown on the right of the image). Oriented structures can be seen clearly and indicated by the arrows in the inset (the zoom-in image of the local area of the scaffold). $\mathrm{X}$-ray micro-computed tomography $(\mu \mathrm{CT})$ images of a freeze cast $60 \mathrm{wt} \%$ organic hybrid scaffold fabricated at a cooling rate of $10{ }^{\circ} \mathrm{C} \mathrm{min}{ }^{-1}(4060 \mathrm{GC} 4$ $10^{\circ} \mathrm{C} \mathrm{min}^{-1}$ ), which show the typical microstructures (b) perpendicular and (c) parallel to the freezing direction. 
FC chitosan $5{ }^{\circ} \mathrm{C} / \mathrm{min}$

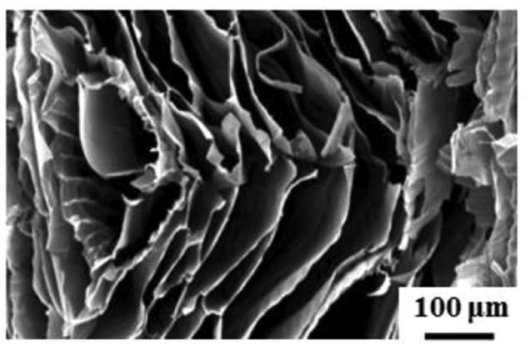

$4060 \mathrm{GC} 45^{\circ} \mathrm{C} / \mathrm{min}$

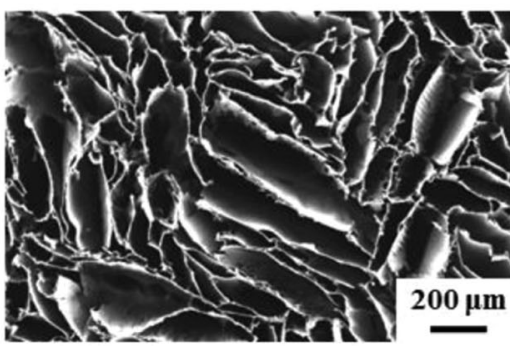

$5050 \mathrm{GC} 45{ }^{\circ} \mathrm{C} / \mathrm{min}$

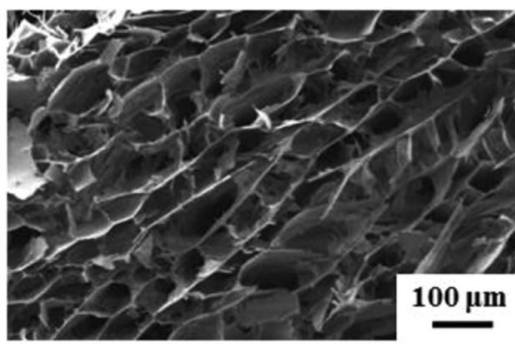

$6040 \mathrm{GC} 410{ }^{\circ} \mathrm{C} / \mathrm{min}$

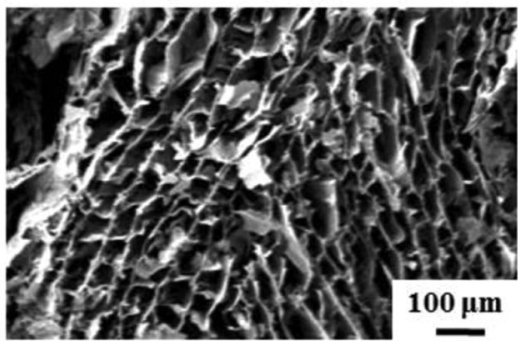

$4060 \mathrm{GC} 110^{\circ} \mathrm{C} / \mathrm{min}$

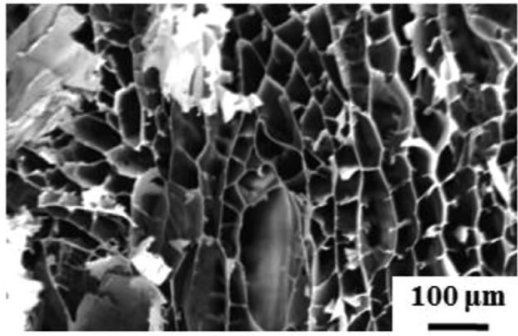

FC chitosan $10^{\circ} \mathrm{C} / \mathrm{min}$

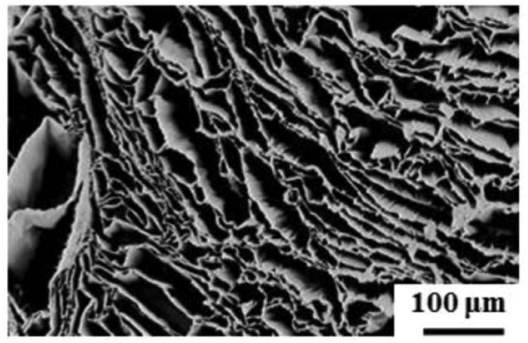

$4060 \mathrm{GC} 410{ }^{\circ} \mathrm{C} / \mathrm{min}$

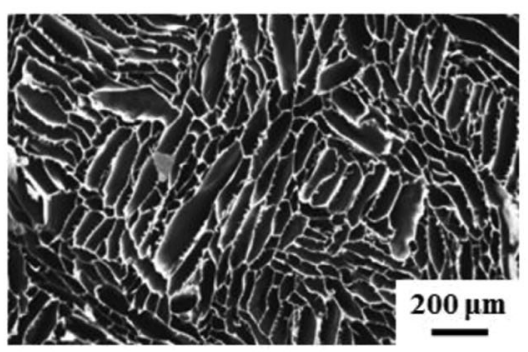

$5050 \mathrm{GC} 410{ }^{\circ} \mathrm{C} / \mathrm{min}$

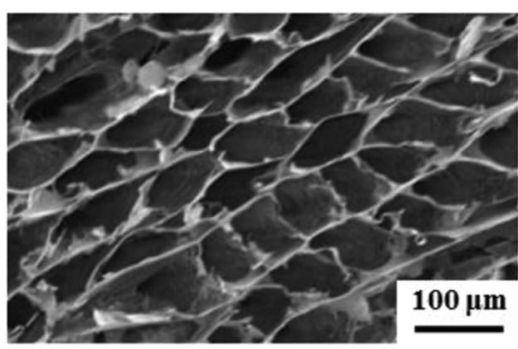

$4060 \mathrm{GC} 210{ }^{\circ} \mathrm{C} / \mathrm{min}$

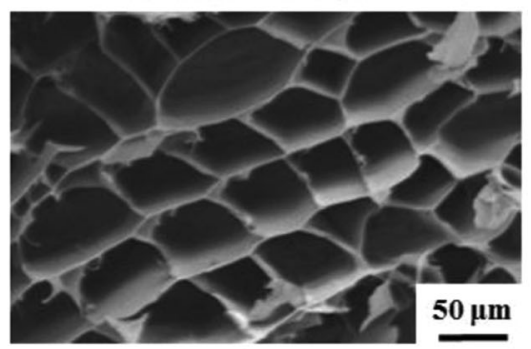

Fig. 6 SEM images of freeze cast scaffolds fabricated with various compositions and cooling rates. Cross-sections perpendicular to the freezing direction, which show the cellular pore structures.

However, with the increase of silica and GPTMS the nucleation and growth of ice crystals changed. High amount of silica and GPTMS or the formation of a condensed silica network disrupted the ice crystallization, reducing the length of the growing ice crystals, in similar manner that glycerol does when added into a solution for freeze casting. ${ }^{45}$ The pore morphology then changed from more lamellar for FC chitosan to more tubelike as the content of silica and GPTMS increased. ${ }^{46}$ 
FC chitosan $5{ }^{\circ} \mathrm{C} / \mathrm{min}$

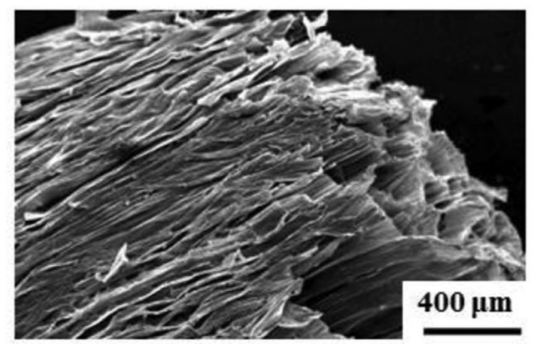

$4060 \mathrm{GC4} 5{ }^{\circ} \mathrm{C} / \mathrm{min}$

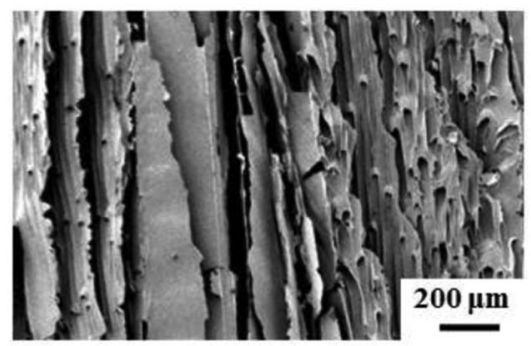

$5050 \mathrm{GC} 45{ }^{\circ} \mathrm{C} / \mathrm{min}$

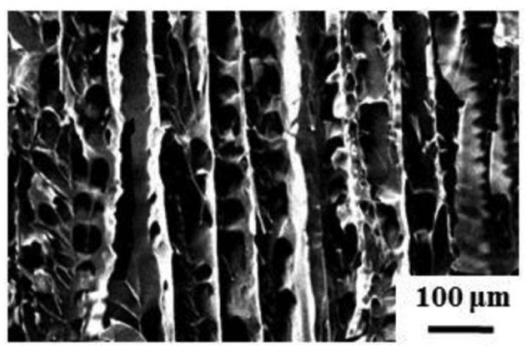

$6040 \mathrm{GC} 410{ }^{\circ} \mathrm{C} / \mathrm{min}$

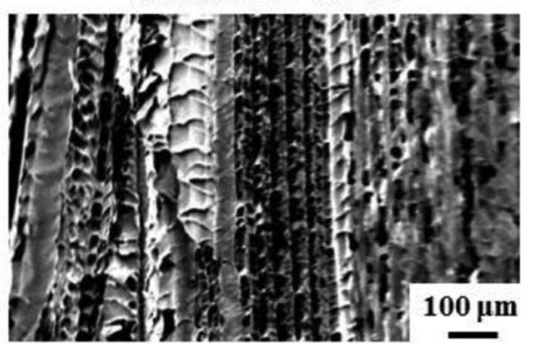

$4060 \mathrm{GC} 110{ }^{\circ} \mathrm{C} / \mathrm{min}$

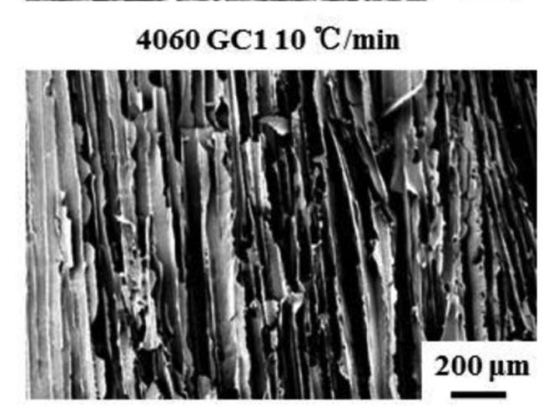

FC chitosan $10{ }^{\circ} \mathrm{C} / \mathrm{min}$

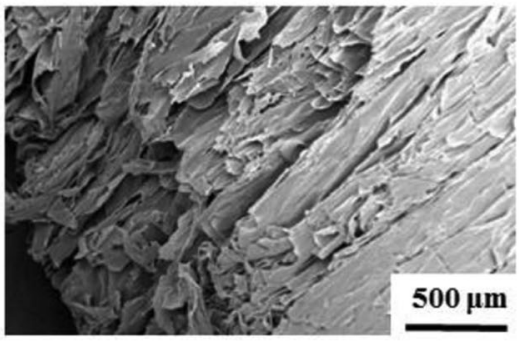

$4060 \mathrm{GC} 410{ }^{\circ} \mathrm{C} / \mathrm{min}$

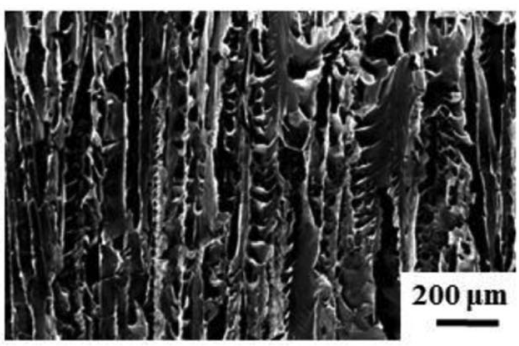

$5050 \mathrm{GC} 410{ }^{\circ} \mathrm{C} / \mathrm{min}$

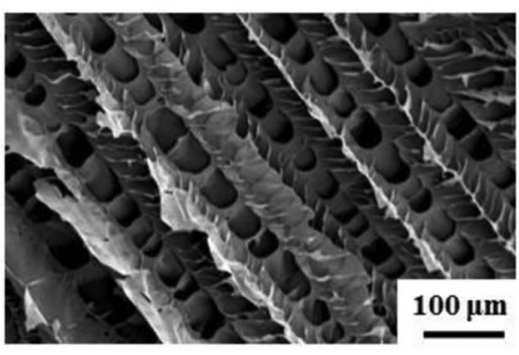

$4060 \mathrm{GC} 210^{\circ} \mathrm{C} / \mathrm{min}$

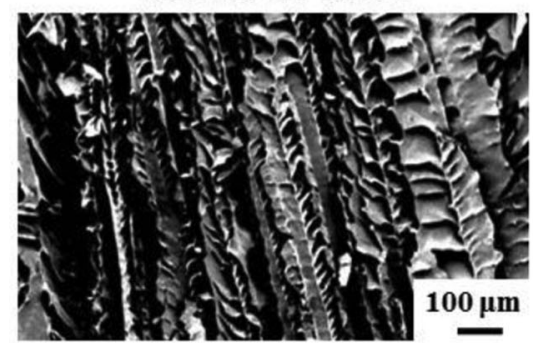

Fig. 7 SEM images of freeze cast scaffolds fabricated with various compositions and cooling rates. Cross-sections parallel to the freezing direction, which show the oriented pore structures.

The influence of cooling rate on scaffold structure was also investigated. Fig. 6 compared the freeze cast scaffolds (i.e. FC chitosan, 4060 GC4 and 5050 GC4) fabricated at the cooling rates $5{ }^{\circ} \mathrm{C} \mathrm{min}^{-1}$ and $10{ }^{\circ} \mathrm{C} \mathrm{min}^{-1}$. Increasing the cooling velocity resulted in smaller pore size for $4060 \mathrm{GC} 4$ and FC chitosan since, which is in accordance with literature. ${ }^{25,27}$ Regarding the 5050 hybrids pore size was surprisingly similar in both scaffolds using different cooling rates $(\sim 50 \mu \mathrm{m}$ along the long axis). This could 
be explained by the disruption of the silica network inhibiting the growth of ice crystal having more effect than the change in cooling rate at such high inorganic content.

An ideal scaffold for cartilage regeneration would be pressfit into defects. The scaffold stiffness should match that of the cartilage so the scaffold can transfer load (mechanotransduction) to cells and it should have a pore network that provokes radial ingrowth. Interconnect sizes should be lower than bone scaffolds as vascularization is not needed, and cell-cell interactions are important. Pore sizes are likely to be $\sim 60 \mu \mathrm{m}$ with interconnect sizes of $\sim 20 \mu \mathrm{m}$ (similar to the cell size) while maintaining high connectivity. ${ }^{9}$

Fig. 7 shows SEM images of cross-sections of the scaffolds in the directions parallel to the freezing direction. Highly ordered morphology can be observed for all the freeze cast scaffolds. However differences can also be seen. The lamellae in FC chitosan were tightly packed in this direction, which could be due to the highly flexibility of material so that compression occurred easily during the cutting procedure for SEM imaging. The compressed structures did not recover after compression and cutting. For freeze cast hybrid scaffolds, morphology can be categorized into two types, i.e. flat lamellar sheet (e.g. $4060 \mathrm{GC} 4$ and $4060 \mathrm{GC} 110^{\circ} \mathrm{C} \mathrm{min}^{-1}$ ) and fishbone like structure with interconnected oval pores embedded into the scaffold struts (e.g. $5050 \mathrm{GC} 4$ and $6040 \mathrm{GC} 410{ }^{\circ} \mathrm{C} \mathrm{min}{ }^{-1}$ ). However, these two different shapes did not occur with any specific trend of hybrid composition. Lamellar-sheet like morphology was observed in all the scaffolds. Fig. S4 (ESI $\dagger$ ) indicates this occurrence in 4060 and $5050 \mathrm{GC} 410{ }^{\circ} \mathrm{C} \mathrm{min}{ }^{-1}$. Here it is proposed that the lamellar-sheet like shape is what the oriented structure should really be, which is generated when the ice crystals grow steadily during freezing. Whereas the fish-bone like microstructure with embedded pores is formed due to the unstable ice crystal growth at the early stage of solidification. Cooney et al. demonstrated that in an aqueous chitosan system the formation of oval pores is more likely to occur at the higher temperature $\left(0\right.$ to $-50{ }^{\circ} \mathrm{C}$ ) and if the temperature gradient is high, the oval ice crystals can evolve into lamellar structures. ${ }^{47}$ This can be further verified by the $\mu \mathrm{CT}$ image of $4060 \mathrm{GC} 410{ }^{\circ} \mathrm{C} \mathrm{min}^{-1}$ (Fig. S4c, ESI $\dagger$ ), of which the mid upper part was scanned. In this position the temperature was low during the freeze casting process, thus the lamellar microstructures without oval pores can only be observed.

For tissue regeneration, a scaffold with high porosity is essential since it can facilitate the exchange of nutrient, oxygen, metabolic wastes and molecular signalling and thus cell proliferation. Here porosity measurement was carried out for all the freeze cast scaffolds and was given in Table 4 . A mean of $97 \%$ porosity was found and the differences were minor between compositions. Therefore, in these silica/chitosan hybrid systems porosity mainly depended on the water content in solution. Such extremely high porosity can compromise mechanical properties.

\subsection{Evaluation of anisotropic compression behaviour}

Anisotropic microstructures can result in anisotropic mechanical response, which is the major characteristic of freeze cast scaffolds. Higher compressive strength along the freezing direction, compared to the direction perpendicular to the freezing
Table 4 Porosity of freeze cast scaffolds fabricated at a cooling rate of $10{ }^{\circ} \mathrm{C} \mathrm{min}^{-1}$ with various compositions. Values were obtain from the bulk density (mean values \pm standard deviation, $n=5$ ) and the skeletal density (mean values \pm standard deviation, $n=3$ ) of the scaffolds

\begin{tabular}{llll}
\hline Sample & $\begin{array}{l}\text { Bulk density } \\
\left(\mathrm{g} \mathrm{cm}^{-3}\right)\end{array}$ & $\begin{array}{l}\text { Skeletal density } \\
\left(\mathrm{g} \mathrm{cm}^{-3}\right)\end{array}$ & $\begin{array}{l}\text { Porosity } \\
(\%)\end{array}$ \\
\hline $4060 \mathrm{GC} 1$ & $0.056 \pm 0.005$ & $1.716 \pm 0.005$ & 96.71 \\
$4060 \mathrm{GC} 2$ & $0.050 \pm 0.002$ & $1.622 \pm 0.014$ & 96.95 \\
$4060 \mathrm{GC} 4$ & $0.044 \pm 0.001$ & $1.694 \pm 0.005$ & 97.42 \\
5050 GC4 & $0.055 \pm 0.001$ & $1.725 \pm 0.018$ & 96.82 \\
6040 GC4 & $0.069 \pm 0.002$ & $1.773 \pm 0.005$ & 96.13 \\
FC chitosan & $0.039 \pm 0.003$ & $1.653 \pm 0.014$ & 97.62
\end{tabular}

direction, was expected due to the highly ordered scaffold structures, which is promising for the application in tissue defects that strong and tough mechanical properties dominant in one direction are required.

In this work silica/chitosan hybrid scaffolds fabricated by the freeze casting technique were shown to have anisotropic microstructures; therefore, compression testing was carried out to investigate the corresponding anisotropic mechanical behaviours in both directions. The effects of silica content, the GPTMS cross-linking degree and the cooling rate on mechanical properties were also examined.

The mechanical properties of the scaffolds and the relevant data are presented in Fig. 8a and Table 5. FC chitosan showed a response typical of elastic-plastic polymer foams when compressed parallel to the freezing direction. Hybrids produced stress/stain curves that were typical of materials with elasticbrittle deformation behaviour: a plateau that is not very flat with "noise" corresponding to small fracture events. The compressive strengths at the yield point (Fig. 8a-ii) of the hybrids were higher compared to the pure chitosan scaffold (FC chitosan), which had an average value of $\sim 36 \mathrm{kPa}$ as (Table 5). As expected, increasing organic content from $40 \%$ to $60 \%$ reduced the yield strength from $184 \mathrm{kPa}$ to $123 \mathrm{kPa}$ (at GC4, $10{ }^{\circ} \mathrm{C} \mathrm{min}{ }^{-1}$ cooling). Increasing the amount of GPTMS from GC4 to GC1 $\left(60 \%\right.$ organic, $10{ }^{\circ} \mathrm{C} \mathrm{min}{ }^{-1}$ cooling) increased the yield strength from $123 \mathrm{kPa}$ to $200 \mathrm{kPa}$. This could be attributed to the formation of a more condensed silica network in higher GPTMS hybrids and more silica introduced into the scaffold to reduce flexibility in higher inorganic hybrid. However changes in strain to yield and stiffness were not clear. Adjusting the cooling rate also showed minor effects on the compressive strength (Fig. 8a-ii and Table 5). The small difference could be due to the joint action of the very high porosities of the scaffolds ( $\sim 97 \%)$, thin pore struts and the high flexibility induced by chitosan. The compressive modulus was approximately $8 \mathrm{MPa}$ for all the hybrid scaffolds (Fig. 8a-iii and Table 5).

The hybrid scaffolds fabricated by the freeze casting technique have improved mechanical properties compared to those obtained by freeze-drying (compressive strength $0.06-0.14 \mathrm{MPa}$ ) ${ }^{20}{ }^{2}$ Freeze cast hybrid scaffolds may be promising for anisotropic soft tissue regeneration such as cartilage due to anisotropic structures.

When the freeze cast scaffolds were compressed perpendicular to the freezing direction, a totally different mechanical response occurred. The stress-strain curves (Fig. 8b) show that in this direction all the scaffolds exhibited a compressive 
(a)
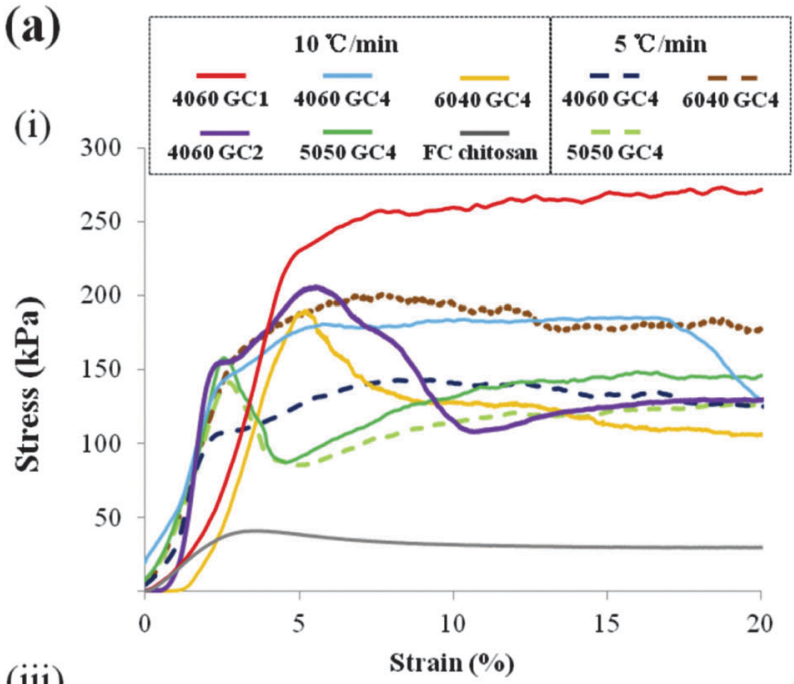

(ii)

(iii)

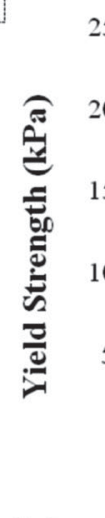

(iv)
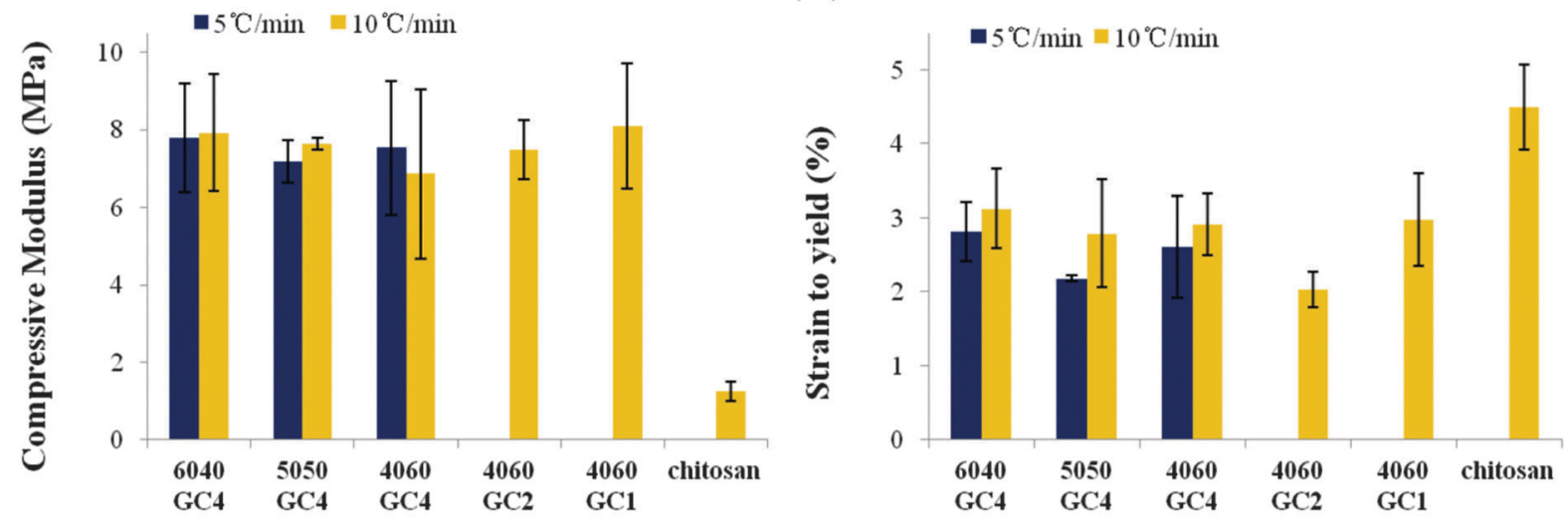

(b)

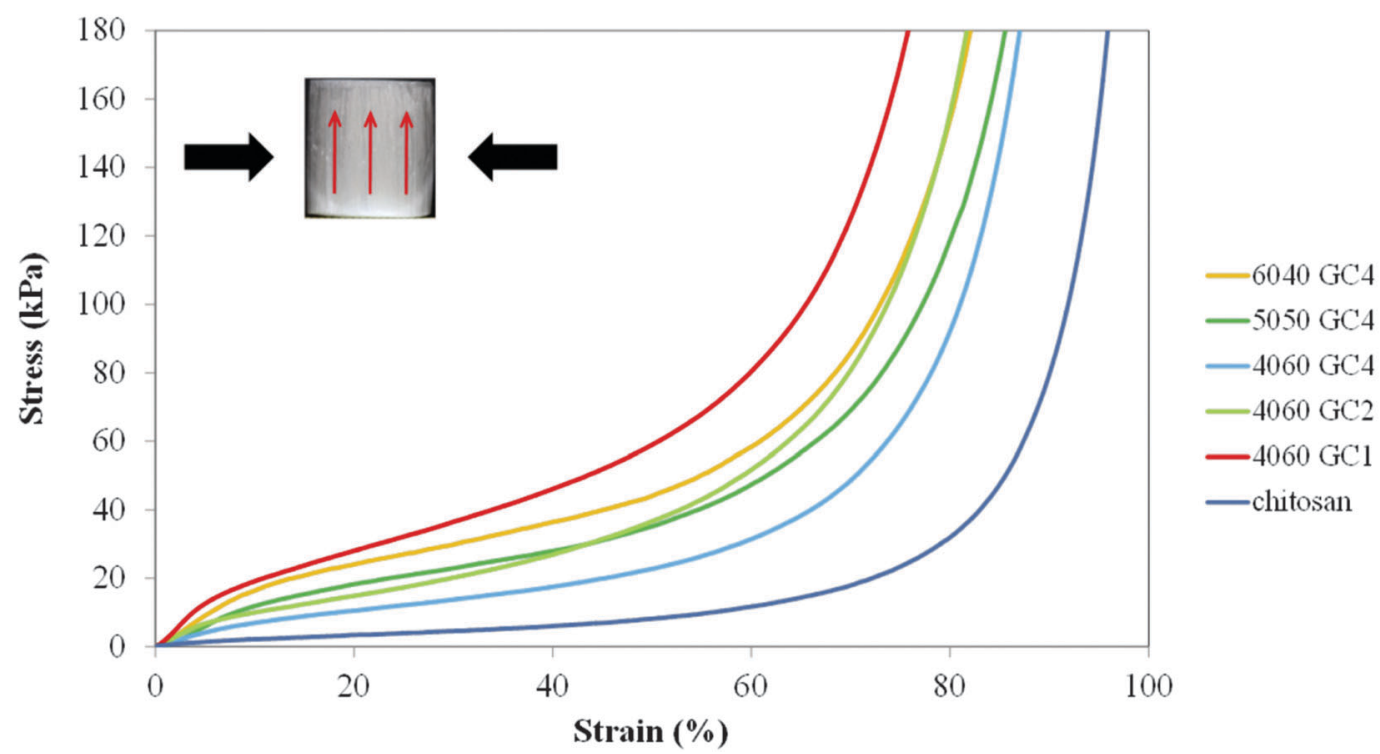

Fig. 8 (a) Compression testing for freeze cast scaffolds fabricated with various cooling rates and compositions, which was carried out along the freezing direction and was shown by the inset in (ii), the red arrows indicate the freezing direction: (i) representative stress-strain curves for different compressed scaffolds. Comparison of the critical mechanical properties between various freeze cast scaffolds with (ii) compressive strength at the yield point, (iii) compressive modulus and (iv) strain at the yield point $(n=5)$. (b) Stress-strain curves for freeze cast scaffolds fabricated at a cooling rate of $10{ }^{\circ} \mathrm{C} \mathrm{min}^{-1}$ with various compositions. Compression was carried out perpendicular to the freezing direction (shown by the inset, the red arrows indicate the freezing direction). All the compressed scaffolds showed an elastic behaviour in this direction but stiffness increased as GPTMS or inorganic content were increased. 
Table 5 Mechanical properties obtained from the compression testing for freeze cast scaffolds fabricated with various cooling rates and compositions (loaded parallel to the freezing direction), mean values \pm standard deviation $(n=5)$

\begin{tabular}{lclll}
\hline Sample & $\begin{array}{l}\text { Cooling rate } \\
\left({ }^{\circ} \mathrm{C} \mathrm{min}^{-1}\right)\end{array}$ & $\begin{array}{l}\text { Strain to } \\
\text { yield }(\%)\end{array}$ & $\begin{array}{l}\text { Yield strength } \\
(\mathrm{kPa})\end{array}$ & $\begin{array}{l}\text { Compressive } \\
\text { modulus }(\mathrm{MPa})\end{array}$ \\
\hline $6040 \mathrm{GC} 4$ & 5 & $2.8 \pm 0.4$ & $162.2 \pm 24.4$ & $7.8 \pm 1.4$ \\
& 10 & $3.1 \pm 0.5$ & $184.0 \pm 3.7$ & $7.9 \pm 1.5$ \\
$5050 \mathrm{GC} 4$ & 5 & $2.2 \pm 0.1$ & $129.8 \pm 4.6$ & $7.2 \pm 0.5$ \\
& 10 & $2.8 \pm 0.7$ & $167.8 \pm 26.9$ & $7.6 \pm 0.1$ \\
$4060 \mathrm{GC} 4$ & 5 & $2.6 \pm 0.7$ & $113.3 \pm 20.9$ & $7.5 \pm 1.7$ \\
& 10 & $2.9 \pm 0.4$ & $122.8 \pm 12.0$ & $6.9 \pm 2.2$ \\
4060 GC2 & 10 & $2.0 \pm 0.2$ & $163.6 \pm 24.8$ & $7.5 \pm 0.8$ \\
4060 GC1 & 10 & $3.0 \pm 0.6$ & $200.8 \pm 15.8$ & $8.1 \pm 1.6$ \\
FC chitosan & 10 & $4.5 \pm 0.6$ & $36.5 \pm 5.9$ & $1.3 \pm 0.2$ \\
\hline
\end{tabular}

behaviour comparable to an elastomeric foam and were highly flexible. These properties may be suitable for applications in soft tissue defects where many low load cycles occur. ${ }^{11}$ In addition, the flexibility in this direction could be tailored by varying the chitosan content and the GPTMS amount, i.e. the scaffold became stiffer with increasing the silica (for a given GC) and the coupling degree from GPTMS (for a given inorganic/organic weight ratio). This suggests that the oriented structures play a dominant role in the scaffold stiffness.

Regarding the $60 \mathrm{wt} \%$ organic hybrid scaffolds (4060 GC4), high elasticity was observed perpendicular to the freezing direction (Fig. 9a). In this direction the scaffold can recover to the original shape even after large compressions, e.g. $60 \%$. By comparison when the force was applied along the freezing direction (Fig. 9b), the scaffold structure ruptured and could not recover after being released from the compressor. To our knowledge, this property of being stiff in one direction and elastic in the perpendicular direction is unique and could be due to orientation in the molecular structure of the hybrid along the ice crystal/pore walls, due to the chitosan. Such unique property is attractive for surgical treatment since it may facilitate squeezing the scaffold into defect sites by surgeons from the flexible and elastic side and meanwhile provides mechanical support from the stiff direction, which is promising for tissue regeneration.

\section{Conclusions}

Silica/chitosan hybrid scaffolds were successfully fabricated with the combination of the sol-gel process and the freeze casting technique. The scaffolds were demonstrated as true class II hybrids with covalent cross-linking between the chitosan and the silica network through the primary amine of chitosan and the epoxide ring of the coupling agent GPTMS. However, much of the GPTMS formed diols, which did not bond to the chitosan. The inorganic/organic cross-linking degree and the dissolution rate can be tailored by varying the GPTMS content, i.e. increasing the GPTMS amount resulted in the enhancement of inorganic/organic coupling and a reduction in the dissolution of the silica network. The hybrids were homogeneous throughout the bulk, suggesting a well dispersion and integrity of the chitosan and silica components.

Highly ordered lamellar pore structures in the scaffolds were obtained using the unidirectional freeze casting process. A cellular morphology was observed perpendicular to the freezing direction. Larger pore sizes can be obtained by decreasing the cooling velocity, the silica and the GPTMS quantities, showing the tailorable properties of the hybrid scaffolds. A mean porosity of $97 \%$ was also achieved due to the low chitosan concentration $\left(17 \mathrm{mg} \mathrm{mL}^{-1}\right.$ ) during the preparation of the hybrid sol. Such high porosity caused low compressive strengths in the freeze cast hybrid scaffolds in the range of $150-250 \mathrm{kPa}$ along the freezing direction. However, the anisotropic structures could be attractive to anisotropic tissue regeneration such as sub-articular cartilage. In addition, the unique highly elastic property in high organic hybrids (e.g. 4060) when loaded perpendicularly to the freezing direction may bring more convenient surgical implantation by easily squeezing and pressing the scaffold into the tissue defect from this direction without deteriorating the mechanical properties from the strong side, which is encouraging for tissue therapy.

\section{Acknowledgements}

The authors wish to thank EPSRC (EP/I020861/1) for funding and providing space at the Research Complex at Harwell via

(a)

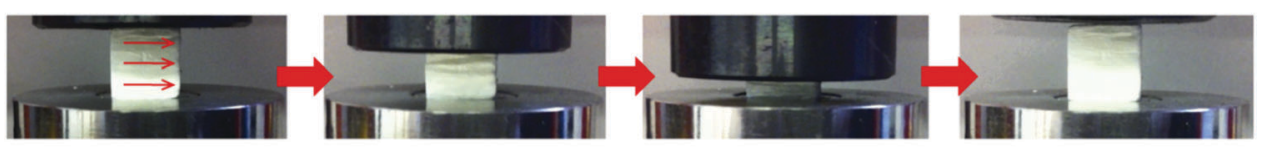

(b)

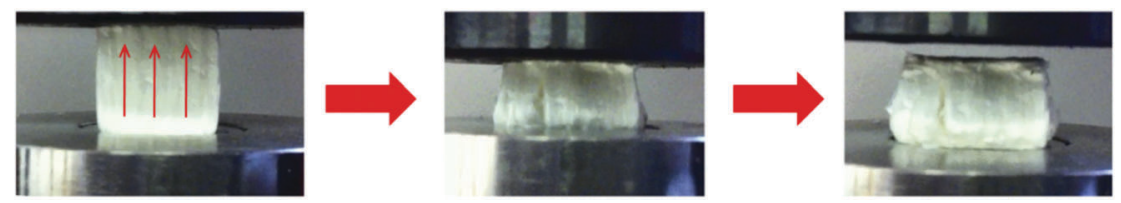

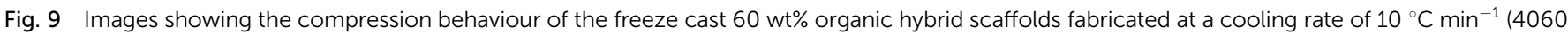

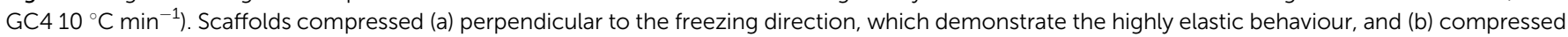

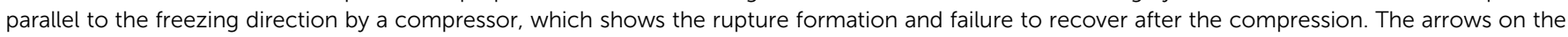
sample indicate the freezing direction. 
EP/I02249X/1 and the Diamond Light Source for beamtime. J.V.H. thanks the EPSRC and the University of Warwick for partial funding of the solid-state NMR infrastructure at Warwick, and acknowledge additional support for this infrastructure obtained through Birmingham Science City: Innovative Uses for Advanced Materials in the Modern World (West Midlands Centre for Advanced Materials Projects 1 and 2), with support from Advantage West Midlands (AWM) and partial funding by the European Regional Development Fund (ERDF). E.S. and C.W. would like to thank the Engineering and Physical Sciences Research Council (EPSRC) of the UK for funding through grant EP/F033605/1.

\section{References}

1 The U.S. Department of Health and Human Services, 2020: A New Vision - A Future for Regenerative Medicine, 2006.

2 M. M. Stevens and J. H. George, Exploring and engineering the cell surface interface, Science, 2005, 310, 1135-1138.

3 M. N. Rahaman, D. E. Day, B. S. Bal, Q. Fu, S. B. Jung, L. F. Bonewald and A. P. Tomsia, Bioactive glass in tissue engineering, Acta Biomater., 2011, 7, 2355-2373.

4 J. R. Jones, Review of bioactive glass: From Hench to hybrids, Acta Biomater., 2013, 9, 4457-4486.

5 J. R. Jones, L. M. Ehrenfried and L. L. Hench, Optimising bioactive glass scaffolds for bone tissue engineering, Biomaterials, 2006, 27, 964-973.

6 Q. Fu, E. Saiz and A. P. Tomsia, Bioinspired strong and highly porous glass scaffolds, Adv. Funct. Mater., 2011, 21, 1058-1063.

7 K. Rezwan, Q. Z. Chen, J. J. Blaker and A. R. Boccaccini, Biodegradable and bioactive porous polymer/inorganic composite scaffolds for bone tissue engineering, Biomaterials, 2006, 27, 3413-3431.

8 J. J. Blaker, A. Bismarck, A. R. Boccaccini, A. M. Young and S. N. Nazhat, Premature degradation of $\operatorname{poly}(\alpha$-hydroxyesters $)$ during thermal processing of Bioglass ${ }^{\mathbb{R}}$-containing composites, Acta Biomater., 2010, 6, 756-762.

9 G. Filardo, E. Kon, A. Roffi, A. Di Martino and M. Marcacci, Scaffold-based repair for cartilage healing: a systematic review and technical note, Arthroscopy: The Journal of Arthroscopic \& Related Surgery, 2013, 29, 174-186.

10 K.-Y. Saw, A. Anz, S. Merican, Y.-G. Tay, K. Ragavanaidu, C. S. Jee and D. A. McGuire, Articular cartilage regeneration with autologous peripheral blood progenitor cells and hyaluronic acid after arthroscopic subchondral drilling: a report of 5 cases with histology, Arthroscopy: The Journal of Arthroscopic \& Related Surgery, 2011, 27, 493-506.

11 O. Mahony, O. Tsigkou, C. Ionescu, C. Minelli, L. Ling, R. Hanly, M. E. Smith, M. M. Stevens and J. R. Jones, SilicaGelatin Hybrids with Tailorable Degradation and Mechanical Properties for Tissue Regeneration, Adv. Funct. Mater., 2010, 20, 3835-3845.

12 B. M. Novak, Hybrid nanocomposite materials - between inorganic glasses and organic polymers, Adv. Mater., 1993, 5, 422-433.
13 D. Tian, S. Blacher, J. P. Pirard and R. Jerome, Biodegradable and biocompatible inorganic-organic hybrid materials. 3. A valuable route to the control of the silica porosity, Langmuir, 1998, 14, 1905-1910.

14 M. N. Kumar, R. A. Muzzarelli, C. Muzzarelli, H. Sashiwa and A. J. Domb, Chitosan chemistry and pharmaceutical perspectives, Chem. Rev., 2004, 104, 6017-6084.

15 P. Agrawal, G. J. Strijkers and K. Nicolay, Chitosan-based systems for molecular imaging, Adv. Drug Delivery Rev., 2010, 62, 42-58.

16 Y. Shirosaki, K. Tsuru, S. Hayakawa, A. Osaka, M. A. Lopes, J. D. Santos and M. H. Fernandes, In vitro cytocompatibility of MG63 cells on chitosan-organosiloxane hybrid membranes, Biomaterials, 2005, 26, 485-493.

17 Y. Shirosaki, T. Okayama, K. Tsuru, S. Hayakawa and A. Osaka, Synthesis and cytocompatibility of porous chitosan-silicate hybrids for tissue engineering scaffold application, Chem. Eng. J., 2008, 137, 122-128.

18 Y. Shirosaki, K. Tsuru, S. Hayakawa, A. Osaka, M. A. Lopes, J. D. Santos, M. A. Costa and M. H. Fernandes, Physical, chemical and in vitro biological profile of chitosan hybrid membrane as a function of organosiloxane concentration, Acta Biomater., 2009, 5, 346-355.

19 Y. Shirosaki, K. Tsuru, H. Moribayashi, S. Hayakawa, Y. Nakamura, I. R. Gibson and A. Osaka, Preparation of osteocompatible $\mathrm{Si}(\mathrm{IV})$-enriched chitosan-silicate hybrids, J. Ceram. Soc. Jpn., 2010, 118, 989-992.

20 L. S. Connell, F. Romer, M. Suarez, E. M. Valliant, Z. Y. Zhang, P. D. Lee, M. E. Smith, J. V. Hanna and J. R. Jones, Chemical characterisation and fabrication of chitosan-silica hybrid scaffolds with 3-glycidoxypropyl trimethoxysilane, J. Mater. Chem. B, 2014, 2, 668-680.

21 L. Gabrielli, L. Connell, L. Russo, J. Jimenez-Barbero, F. Nicotra, L. Cipolla and J. R. Jones, Exploring GPTMS reactivity against simple nucleophiles: chemistry beyond hybrid materials fabrication, $R S C A d v ., 2014$, 4, 1841-1848.

22 G. Poologasundarampillai, C. Ionescu, O. Tsigkou, M. Murugesan, R. G. Hill, M. M. Stevens, J. V. Hanna, M. E. Smith and J. R. Jones, Synthesis of bioactive class II poly(gamma-glutamic acid)/silica hybrids for bone regeneration, J. Mater. Chem., 2010, 20, 8952-8961.

23 E. M. Valliant, F. Romer, D. Wang, D. S. McPhail, M. E. Smith, J. V. Hanna and J. R. Jones, Bioactivity in silica/ poly(gamma-glutamic acid) sol-gel hybrids through calcium chelation, Acta Biomater., 2013, 9, 7662-7671.

24 S. Deville, Freeze-Casting of Porous Biomaterials: Structure, Properties and Opportunities, Materials, 2010, 3, 1913-1927.

25 S. Deville, Freeze-Casting of Porous Ceramics: A Review of Current Achievements and Issues, Adv. Eng. Mater., 2008, 10, 155-169.

26 S. Deville, E. Saiz, R. K. Nalla and A. P. Tomsia, Freezing as a path to build complex composites, Science, 2006, 311, 515-518.

27 U. G. Wegst, M. Schecter, A. E. Donius and P. M. Hunger, Biomaterials by freeze casting, Philos. Trans. R. Soc., A, 2010, 368, 2099-2121. 
28 H. F. Zhang, I. Hussain, M. Brust, M. F. Butler, S. P. Rannard and A. I. Cooper, Aligned two- and threedimensional structures by directional freezing of polymers and nanoparticles, Nat. Mater., 2005, 4, 787-793.

29 W. L. Li, K. Lu and J. Y. Walz, Freeze casting of porous materials: review of critical factors in microstructure evolution, Int. Mater. Rev., 2012, 57, 37-60.

30 M. Pourhaghgouy and A. Zamanian, Physical and Mechanical Properties of the Fully Interconnected Chitosan IceTemplated Scaffolds, J. Appl. Polym. Sci., 2015, 132, 7.

31 A. L. B. Maçon, T. B. Kim, E. M. Valliant, K. Goetschius, R. K. Brow, D. E. Day, A. Hoppe, A. R. Boccaccini, I. Y. Kim, C. Ohtsuki, T. Kokubo, A. Osaka, M. Vallet-Regi, D. Arcos, L. Fraile, A. J. Salinas, A. V. Teixeira, Y. Vueva, R. M. Almeida, M. Miola, C. Vitale-Brovarone, E. Verne, W. Hoeland and J. R. Jones, A unified in vitro evaluation for apatite-forming ability of bioactive glasses and their variants, J. Mater. Sci.: Mater. Med., 2015, 26, 115.

32 J. R. Jones, G. Poologasundarampillai, R. C. Atwood, D. Bernard and P. D. Lee, Non-destructive quantitative 3D analysis for the optimisation of tissue scaffolds, Biomaterials, 2007, 28, 1404-1413.

33 C. Rau, U. Wagner, Z. Pešić and A. De Fanis, Coherent imaging at the Diamond beamline I13, Phys. Status Solidi A, 2011, 208, 2522-2525.

34 V. Titarenko, S. Titarenko, P. J. Withers, F. De Carlo and $\mathrm{X}$. Xiao, Improved tomographic reconstructions using adaptive time-dependent intensity normalization, J. Synchrotron Radiat., 2010, 17, 689-699.

35 D. Carboni, A. Pinna, H. Amenitsch, M. F. Casula, D. Loche, L. Malfatti and P. Innocenzi, Getting order in mesostructured thin films, from pore organization to crystalline walls, the case of 3-glycidoxypropyltrimethoxysilane, Phys. Chem. Chem. Phys., 2015, 17, 10679-10686.

36 B. Alonso, D. Massiot, F. Babonneau, G. Brusatin, G. D. Giustina, T. Kidchob and P. Innocenzi, Structural control in germania hybrid organic-inorganic materials, Chem. Mater., 2005, 17, 3172-3180.
37 L. Gabrielli, L. Russo, A. Poveda, J. R. Jones, F. Nicotra, J. Jiménez-Barbero and L. Cipolla, Epoxide Opening versus Silica Condensation during Sol-Gel Hybrid Biomaterial Synthesis, Chem. - Eur. J., 2013, 19, 7856-7864.

38 M. Templin, U. Wiesner and H. W. Spiesss, Multinuclear solidstate-NMR studies of hybrid organic-inorganic materials, $A d v$. Mater., 1997, 9, 814-817.

39 K. J. MacKenzie and M. E. Smith, Multinuclear solid-state nuclear magnetic resonance of inorganic materials, Elsevier, 2002.

40 S.-H. Lim and S. M. Hudson, Synthesis and antimicrobial activity of a water-soluble chitosan derivative with a fiberreactive group, Carbohydr. Res., 2004, 339, 313-319.

41 J. Kumirska, M. Czerwicka, Z. Kaczyński, A. Bychowska, K. Brzozowski, J. Thöming and P. Stepnowski, Application of spectroscopic methods for structural analysis of chitin and chitosan, Mar. Drugs, 2010, 8, 1567-1636.

42 D. Depan, B. Kumar and R. P. Singh, Preparation and characterization of novel hybrid of chitosan-g-PDMS and sodium montmorrilonite, J. Biomed. Mater. Res., Part B, 2008, 84, 184-190.

43 D. L. Pavia, G. M. Lampman, G. S. Kriz and J. A. Vyvyan, Introduction to Spectroscopy, Brooks/Cole, Cengage Learning, US, 2009.

44 S. D. McCullen, H. Autefage, A. Callanan, E. Gentleman and M. M. Stevens, Anisotropic Fibrous Scaffolds for Articular Cartilage Regeneration, Tissue Eng., Part A, 2012, 18, 2073-2083.

45 S. W. Sofie and F. Dogan, Freeze Casting of Aqueous Alumina Slurries with Glycerol, J. Am. Ceram. Soc., 2001, 84, 1459-1464.

46 S. R. Mukai, H. Nishihara and H. Tamon, Formation of monolithic silica gel microhoneycombs (SMHs) using pseudosteady state growth of microstructural ice crystals, Chem. Commun., 2004, 874-875.

47 M. J. Cooney, C. Lau, M. Windmeisser, B. Y. Liaw, T. Klotzbach and S. D. Minteer, Design of chitosan gel pore structure: towards enzyme catalyzed flow-through electrodes, J. Mater. Chem., 2008, 18, 667-674. 